This document is the accepted manuscript version of the following article:

Rigby, M., Park, S., Saito, T., Western, L. M., Redington, A. L., Fang, X., ... Young, D.

(2019). Increase in CFC-11 emissions from eastern China based on atmospheric

observations. Nature, 569(7757), 546-550. https://doi.org/10.1038/s41586-019-1193-4

\title{
1 Increase in CFC-11 emissions from eastern China based on atmospheric observations
}

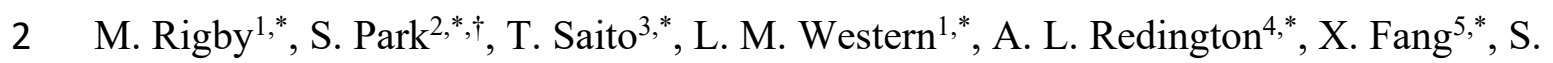

3 Henne $^{6}$, A. J. Manning 4 , R. G. Prinn ${ }^{5}$, G. S. Dutton ${ }^{7}$, P. J. Fraser ${ }^{8}$, A. L. Ganesan ${ }^{9}$, B. D.

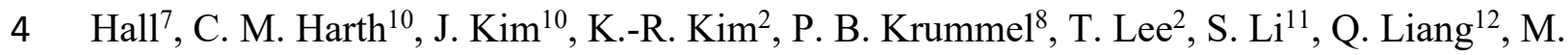

$5 \quad$ F. Lunt ${ }^{13}$, S. A. Montzka ${ }^{7}$, J. Mühle ${ }^{10}$, S. O’Doherty ${ }^{1}$, M.-K. Park ${ }^{11}$, S. Reimann ${ }^{6}$, P. K.

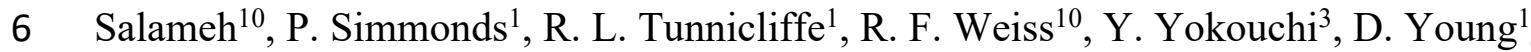

$8{ }^{1}$ School of Chemistry, University of Bristol, Bristol, UK

$9 \quad{ }^{2}$ Department of Oceanography, Kyungpook National University, Daegu, Republic of Korea

$10{ }^{3}$ National Institute for Environmental Studies, Tsukuba, Japan

$11 \quad{ }^{4}$ Hadley Centre, Met Office, Exeter, UK

$12{ }^{5}$ Center for Global Change Science, Massachusetts Institute of Technology, Cambridge,

13 Massachusetts, USA

$14{ }^{6}$ Empa, Swiss Federal Laboratories for Materials Science and Technology, Dübendorf,

15 Switzerland

$16{ }^{7}$ Global Monitoring Division, Earth System Research Laboratory, National Oceanic and

17 Atmospheric Administration, USA

${ }^{8}$ Climate Science Centre, CSIRO Oceans and Atmosphere, Aspendale, Victoria, Australia

${ }^{9}$ School of Geographical Sciences, University of Bristol, Bristol, UK

${ }^{10}$ Scripps Institution of Oceanography, University of California San Diego, La Jolla, USA

${ }^{11}$ Kyungpook Institute of Oceanography, Kyungpook National University, Daegu, Republic

of Korea

${ }^{12}$ NASA Goddard Space Flight Center, Greenbelt, USA 
*These authors contributed equally to this work

†Correspondence should be addressed to sparky@knu.ac.kr

4

5 The recovery of the stratospheric ozone layer relies on the continued decline in the

6 atmospheric concentrations of ozone-depleting gases such as the chlorofluorocarbons ${ }^{1}$.

7 The atmospheric concentration of trichlorofluoromethane (CFC-11), the second most abundant chlorofluorocarbon, has declined substantially since the mid-1990s ${ }^{2}$ A recently reported slowdown in the decline of the atmospheric concentration of CFC-11 after 2012, however, implies that global emissions have increased ${ }^{3,4}$. A concurrent increase in CFC-11 emissions from eastern Asia contributes to the global emission increase, but the location and magnitude of this regional source remain uncertain ${ }^{3}$. Here we use high-frequency atmospheric observations from Gosan, Republic of Korea and Hateruma, Japan, together with global monitoring data and atmospheric chemical transport model simulations to investigate regional CFC-11 emissions from eastern Asia. We find that emissions from eastern mainland China are 7.0 $\pm 3.0 \mathrm{Gg}^{-1} \mathrm{higher}^{-1}$ in 2014 - 2017 compared to 2008 - 2012, and the emissions increase arises primarily around the north-eastern provinces of Shandong and Hebei. This increase accounts for a substantial fraction (about $40-60 \%$, or more) of the global CFC-11 emission rise. We find no evidence for a significant increase in emissions from any other eastern Asian countries or other regions of the world where available data allow for the detection of regional emissions. Attribution of any remaining fraction of the global CFC-11 emission rise to other regions is limited by the sparsity of long-term measurements of sufficient

24 frequency near potentially emissive regions. Multiple considerations suggest that the increase in CFC-11 emissions from eastern mainland China is likely the result of new 
4 Before its global production phase-out in 2010, mandated by the Montreal Protocol on

5 Substances that Deplete the Ozone Layer, CFC-11 was primarily used as an agent for

6 blowing foams that were incorporated into buildings and consumer products. As production

7 declined, it was expected that leakage from banks of CFC-11 stored in these foams would

8 dominate its emissions to the atmosphere. Emissions were projected to decline as these banks

9 depleted ${ }^{1}$. However, atmospheric mole fraction data from the National Oceanic and 10 Atmospheric Administration (NOAA) and the Advanced Global Atmospheric Gases

11 Experiment (AGAGE) show that the rate at which CFC-11 has been declining in the global

production and use, inconsistent with the Montreal Protocol agreement to phase out global chlorofluorocarbon production by 2010. Experiment (AGAGE) show that the rate at which $\mathrm{CFC}-11$ has been declining in the global background atmosphere slowed since approximately 2013, and its interhemispheric mole fraction difference increased ${ }^{3,4}$. Whilst variations in atmospheric circulation may have contributed to the slow-down, an increase in northern hemispheric emissions is required, starting after 2012, to explain the majority of these observed changes ${ }^{3}$.

High-frequency atmospheric CFC-11 mole fraction data from the AGAGE network ${ }^{4}$ and Japanese NationalInstitute for Environmental Studies (NIES) ${ }^{5}$ show signals that are consistent with a continuing decline in CFC-11 emissions from Europe, North America and Australia in recent years, but a rise from eastern Asia since 2012 (Figure 4; Extended Data Figure 10). In these datasets, enhancements above the hemispheric background mole fractions are observed when plumes from nearby sources impinge on the measurement stations. For the AGAGE stations in Europe, the west coast of North America, Australia and more remote locations, the magnitudes of above-baseline events are relatively small and have declined throughout the study period (2008 - 2017, inclusive), indicating that emissions near 
1 these sites have dropped. A decline in emissions from North America is consistent with a

2 recent study carried out using independent NOAA data in the USA through $2014^{6}$. In

3 contrast, observations at Hateruma, Japan (location shown in Extended Data Figure 11) show

4 persistent above-baseline events throughout, which have not declined substantially or may

5 have increased, and data from Gosan, Republic of Korea show a strong increase in the

6 magnitude of these events after 2012 (Figure 4). We focus our analysis on eastern Asia

7 because the existing measurement networks only show an increase in the magnitude of

8 above-baseline events in this region. A qualitative emissions increase from somewhere in this

9 region was also indicated in NOAA data from Hawaii ${ }^{3}$.

11 To quantify emissions from eastern Asian countries, atmospheric observation-based "inverse" or "top-down" estimates were carried out with CFC-11 measurements from Gosan and Hateruma observations using two atmospheric chemical transport models and four

14 statistical methods (see Methods). Previous top-down studies in this region between 2001 and 152012 had broadly confirmed inventory-based ("bottom-up") estimates of declining emissions 16 from China ${ }^{7-12}$, and found comparatively small emissions from Japan and the Korean

17 Peninsula ${ }^{10,13}$. Here, we used the NAME $^{14}$ and FLEXPART ${ }^{15}$ models to simulate the transport of CFC-11 from potential sources to the measurement sites. Emissions were derived

19 from the observed mole fractions using NAME and a hierarchical Bayesian "trans-

20 dimensional" inverse method (denoted NAME-HB) $)^{16}$ and the Bayesian Inversion Technique

21 for Emissions Modelling (NAME-InTEM) ${ }^{17}$. Inversions with FLEXPART used two independent analytical Bayesian frameworks (FLEXPART-MIT ${ }^{18}$ and FLEXPART-Empa ${ }^{19}$ ).

23 Analysis using NAME shows that the measurements from the Gosan and Hateruma stations

24 are sensitive to recent emissions from western Japan, the Korean Peninsula, and a region that we define as "eastern mainland China", which encompasses around $35 \%$ of the Chinese 
1 population and contains the provinces of Anhui, Beijing, Hebei, Jiangsu, Liaoning,

2 Shandong, Shanghai, Tianjin and Zhejiang (Extended Data Figure 11). Hence, emissions

3 totals are subsequently provided here only for these regions of China.

4

5 Between 2008 and 2012 (inclusive), we inferred mean CFC-11 emissions of $6.4 \pm 1.2 \mathrm{Gg} \mathrm{yr}^{-1}$

6 for eastern mainland China (Figure 5, the technique for estimating multi-inversion mean

7 emissions is provided in Methods). The mean derived emission during these years for eastern

8 mainland China is similar to an inventory estimate for the whole of China, when scaled by

9 the fraction of the population that reside in the eastern mainland China region ${ }^{7}$. However,

10 whilst the inventory-based emission estimates for China show a downward trend during this

11 period, emission magnitudes derived from atmospheric observations did not decline

12 significantly. After 2012, the top-down estimates from four different inverse techniques show

13 an emission increase of CFC-11 from eastern mainland China, although the exact timing of

14 the initial rise differs between inversions. Average emissions between 2014 and 2017 were

$15 \quad 13.4 \pm 1.7 \mathrm{Gg} \mathrm{yr}^{-1}$ (for consistency with a previous study, we have omitted 2013 as a

16 transitional year $\left.{ }^{3}\right)$. Therefore, the increase in derived emissions between the periods $2008-$

172012 and $2014-2017$ is $7.0 \pm 3.0 \mathrm{Gg} \mathrm{yr}^{-1}$, or around $110 \%$. Our top-down inversion-based

18 estimate for CFC-11 emissions from eastern mainland China for $2014-2017$ is $8.7 \pm 1.0 \mathrm{Gg}$

$19 \mathrm{yr}^{-1}$ higher than the population-weighted inventory-based estimate during this period ${ }^{7}$. When

20 run forward with our inversion-derived emission estimates, the models reproduce the

21 increasing mole fractions during pollution events at the monitoring sites (Extended Data

22 Figure 12). When using data from either Hateruma or Gosan, a rise in emissions is inferred

23 for eastern mainland China (Extended Data Figure 13). However, the magnitude of the rise is

24 smaller and more uncertain when Hateruma is used alone, likely because measurements at

25 this site are less sensitive to the major emissions sources. The derived emissions are relatively 
1 insensitive to the magnitude or distribution of a priori emissions used in the Bayesian

2 inversion frameworks (Extended Data Figure 14). Emissions derived for western Japan and

3 both countries on the Korean peninsula are substantially smaller than those derived for

4 eastern mainland China, and emission magnitudes derived for Japan and Korea did not

5 change significantly during the study period (Extended Data Figure 15).

6

7 To provide context to these eastern Asian emission increases, we provide an update to the

8 global emission magnitudes ${ }^{3,4}$. Long-term observations of baseline mole fractions, which are

9 representative of air masses that have not been substantially influenced by nearby sources,

10 are used to infer changes in the global emission rate of CFC-1 $11^{3,4}$. Using a two-dimensional

11 model of atmospheric transport and chemistry, and assuming a lifetime of 52 years for CFC-

$1211^{20}$, we find that $64 \pm 2 \mathrm{Gg} \mathrm{yr}^{-1}$ were emitted on average between 2008 and 2012 when

13 observations from either AGAGE or NOAA were used (see Methods; Figure 2). These

14 emissions increased to a mean value of $75 \pm 3 \mathrm{Gg} \mathrm{yr}^{-1}$ or $80 \pm 3 \mathrm{Gg} \mathrm{yr}^{-1}$ for 2014 to 2017 ,

15 using AGAGE or NOAA data, respectively, which represents a rise of $11 \pm 3 \mathrm{Gg} \mathrm{yr}^{-1}(17 \pm 5$

$16 \%)$ or $17 \pm 3 \mathrm{Gg} \mathrm{yr}^{-1}(26 \pm 5 \%)$ above emissions during the $2008-2012$ period. Our update

17 shows that the global emission increase previously reported through $2016^{3,4}$ has persisted

18 through 2017. Consistent with previous studies ${ }^{3,4}$, our simplified global modelling approach

19 assumes that atmospheric transport did not vary interannually and our uncertainty estimates

20 do not account for the potential influence of interannual changes in dynamics on the derived

21 emissions. However, it has been demonstrated that changes in atmospheric transport may

22 have contributed to up to $50 \%$ of the slow-down in the rate of decline in global mole fraction,

23 at least between 2014 and $2016^{3}$. Therefore, this inferred average global emission increase,

24 and those that were previously reported, may over-estimate the true global emissions rise in

25 recent years. 
2 When compared to the AGAGE or NOAA-derived global emissions increase, our estimated

3 rise for eastern mainland China accounts for $64 \pm 32 \%$ or $41 \pm 20 \%$ of the total,

4 respectively. However, given that our global emissions increase may be over-estimated by up

5 to a factor of two, owing to unaccounted-for changes in atmospheric dynamics ${ }^{3}$, the fraction

6 of the global emission increase accounted for by eastern mainland China may actually be

7 substantially higher. Hence, these results demonstrate that increased emissions from eastern

8 mainland China since 2012 can account for a large fraction of the concurrent rise in global

9 emissions. However, we cannot rule out additional, but probably smaller, increases from

10 other regions of the world (e.g. northern, southern and western Asia, Africa and South

11 America), potentially including other parts of China, to which our current measurement

12 networks are insensitive.

14 The spatial distribution of emissions inferred within our eastern mainland China region for

15 the periods $2008-2012$ and $2014-2017$ shows the largest change in or around the provinces

16 of Shandong and Hebei, and to a lesser extent near Shanghai (Figure 6). This change is

17 evident across all four inversion results (Extended Data Figure 16). Due to the effect of

18 atmospheric mixing, and because we used a small number of monitoring stations that are

19 relatively far from the major sources, it is not possible to discern the location of emissions

20 sources at finer scales than provinces, or groups of provinces, in the north-eastern part of

21 China. Therefore, it is unclear whether these results indicate that the new emissions originate

22 from a small number of point sources or are more distributed within these regions.

24 A number of considerations suggest that the increase in emissions from China is unlikely to

25 originate from increased rates of escape from pre-phase-out banks of CFC-11 in existing 
1 foam or chillers. First, the spatial distribution of our derived emissions rise is unrelated to

2 population density (Figure 6), whereas the distribution of banks of foams should be roughly

3 distributed by population. Second, our cumulative emissions from eastern mainland China

4 between 2014 and $2017(56 \pm 7 \mathrm{Gg})$ are similar to an inventory-based estimate of the CFC-11

5 bank for all of China in $2013(54 \mathrm{Gg})^{7}$, or almost three times the population-scaled bank for

6 this region, implying that the bank in 2013 was not large enough to accommodate the

7 emissions that occurred in subsequent years. Third, even given the possibility that the

8 Chinese bank size was underestimated for 2012, the emission increase derived for eastern

9 mainland China would require that the bank release fraction increase by around an order of

10 magnitude over the course of five years, a change that seems highly unlikely (Extended Data

11 Figure 8). To bring about such an increase in release rate from the bank would require new

12 emissions from the disposal and destruction of refrigerators more than ten times higher than

13 recently estimated for the whole of China between 2014 and $2017^{21}$, or a larger and more

14 rapid increase in emissions from the demolition of old buildings than was previously

15 predicted for the entire world over a 20-year period $(2020-2040)^{22}$.

17 On the basis of this reasoning, it is likely that the new emissions of CFC-11 are the result of new production that has not been reported to the UNEP Ozone Secretariat. Further information is required to confirm which processes are responsible for releasing this newly

20 produced CFC-11 to the atmosphere. If consistent with historical usage, it would be expected that emissions have primarily occurred during, or following, foam blowing, rather than

22 directly during production. Therefore, the regions where emissions have been identified in

23 this study are not necessarily the same as the locations where the compound has been

24 produced. It may be expected that new production of CFC-11 should be accompanied by new 25 emissions of carbon tetrachloride $\left(\mathrm{CCl}_{4}\right)$, the starting reagent for producing $\mathrm{CFC}-11$, and 
1 CFC-12 $\left(\mathrm{CF}_{2} \mathrm{Cl}_{2}\right)$, which is typically co-produced with CFC-11. Whilst new emissions of

$2 \mathrm{CCl}_{4}$ have been identified from parts of eastern China in recent years ${ }^{23}$, we do not find

3 evidence for an increase in emissions of CFC-12 (Extended Data Figure 9). Given the

4 different historical uses of CFC-11 and CFC-12 (primarily in foam blowing and refrigeration,

5 respectively), the relationship between production and the subsequent rate of emission may

6 also be very different for these two substances. Therefore, the lack of a detectable increase in

7 CFC-12 emissions does not preclude a recent rise in CFC-11 production.

9 Our results show that new CFC-11 emissions from eastern mainland China explain a 10 substantial fraction of the post-2012 rise in global emissions, and are likely due to new, unreported production and use. It is important to recall that before this period, global emissions had not declined as expected since the early 2000s, and by 2012 were around 10 ${\mathrm{Gg} \mathrm{yr}^{-1} \text { higher than projections }}^{3}$. In contrast to the recent rise, we propose that emissions from eastern mainland China are unlikely to have contributed significantly to this earlier "plateau", because our derived emissions for this region during $2008-2012$ are relatively small $(6.4 \pm$

$161.2 \mathrm{Gg} \mathrm{yr}^{-1}$ ) and consistent with estimated release rates from banks ${ }^{7}$. Other countries, and

17 indeed the rest of China, which are not well observed by the current monitoring networks may also have contributed to the unexpected trends in CFC-11, which have now been ongoing for almost two decades. Further investigation will be required to determine which processes have led to the inferred recent increase in emissions from China (and potentially elsewhere) and the magnitude of any associated, unreported CFC-11 production. Depending on which processes are responsible, the new emissions may represent only a fraction of the total new production, with the remainder contained in more slowly emitting banks (e.g.

24 foams). Ultimately, because nearly all known uses of CFC-11 are dispersive, it is the total 
1 production magnitude that will determine the overall impact of these activities on the ozone

2 layer and its recovery.

3

4 References

5 1. Harris, N. R. P. \& Wuebbles, D. J. Chapter 5: Scenarios and information for policymakers.

6 in Scientific Assessment of Ozone Depletion: 2014416 (World Meteorological

7 Organization).

8 2. Carpenter, L. J. \& Reimann, S. Chapter 1: Ozone-Depleting Substances (ODSs) and Other

9 Gases of Interest to the Montreal Protocol. in Scientific assessment of Ozone depletion:

102014 (World Meteorological Organization, 2014).

11 3. Montzka, S. A. et al. An unexpected and persistent increase in global emissions of ozone12 depleting CFC-11. Nature 557, 413-417 (2018).

13 4. Prinn, R. G. et al. History of chemically and radiatively important atmospheric gases from 14 the Advanced Global Atmospheric Gases Experiment (AGAGE). Earth Syst. Sci. Data 10, $15985-1018(2018)$.

16 5. Yokouchi, Y. et al. High frequency measurements of HFCs at a remote site in east Asia 17 and their implications for Chinese emissions. Geophys. Res. Lett. 33, (2006).

6. Hu, L. et al. Considerable contribution of the Montreal Protocol to declining greenhouse gas emissions from the United States: U.S. CFCs, HCFCs, and HFCs Emissions. Geophys. Res. Lett. (2017). doi:10.1002/2017GL074388

7. Fang, X. et al. Changes in Emissions of Ozone-Depleting Substances from China Due to Implementation of the Montreal Protocol. Environ. Sci. Technol. (2018). doi:10.1021/acs.est.8b01280

8. Palmer, P. I. et al. Eastern Asian emissions of anthropogenic halocarbons deduced from aircraft concentration data. J. Geophys. Res. Atmospheres 108, 4753 (2003). 
1 9. An, X. et al. Estimating emissions of HCFC-22 and CFC-11 in China by atmospheric

2 observations and inverse modeling. Sci. China Chem. 55, 2233-2241 (2012).

3 10. Li, S. et al. Emissions of halogenated compounds in East Asia determined from 4 measurements at Jeju Island, Korea. Environ. Sci. Technol. 45, 5668-75 (2011).

5 11. Kim, J. et al. Regional atmospheric emissions determined from measurements at Jeju

6 Island, Korea : Halogenated compounds from China. Geophys. Res. Lett. 37, L12801

7 (2010).

8 12. Vollmer, M. K. et al. Emissions of ozone-depleting halocarbons from China.

9 Geophys. Res. Lett. 36, L15823 (2009).

10 13. Saito, T. et al. Extraordinary halocarbon emissions initiated by the 2011 Tohoku 11 earthquake. Geophys. Res. Lett. 42, 2500-2507 (2015).

12 14. Manning, A. J., O’Doherty, S., Jones, A. R., Simmonds, P. G. \& Derwent, R. G.

13 Estimating UK methane and nitrous oxide emissions from 1990 to 2007 using an inversion 14 modeling approach. J. Geophys. Res. 116, D02305 (2011).

15 15. Stohl, A., Forster, C., Frank, A., Seibert, P. \& Wotawa, G. Technical note: The 16 Lagrangian particle dispersion model FLEXPART version 6.2. Atmospheric Chem. Phys.

$17 \quad 5,2461-2474(2005)$.

18 16. Lunt, M. F., Rigby, M., Ganesan, A. L. \& Manning, A. J. Estimation of trace gas 19 fluxes with objectively determined basis functions using reversible-jump Markov chain 20 Monte Carlo. Geosci. Model Dev. 9, 3213-3229 (2016).

21 17. Arnold, T. et al. Inverse modelling of CF4 and NF3 emissions in East Asia. Atmospheric Chem. Phys. 18, 13305-13320 (2018).

23 18. Fang, X. et al. Rapid increase in ozone-depleting chloroform emissions from China. Nat. Geosci. (2018). doi:10.1038/s41561-018-0278-2 
1 19. Henne, S. et al. Validation of the Swiss methane emission inventory by atmospheric

2 observations and inverse modelling. Atmospheric Chem. Phys. 16, 3683-3710 (2016).

3 20. Ko, M. K. W., Newman, P. A., Reimann, S. \& Strahan, S. E. Recommended Values

4 for Steady-State Atmospheric Lifetimes and their Uncertainties. in SPARC Report on the

5 Lifetimes of Stratospheric Ozone-Depleting Substances, Their Replacements, and Related

6 Species (eds. Ko, M., Newman, P., Reimann, S. \& Strahan, S.) (Stratospheric Processes

$7 \quad$ And their Role in Climate, 2013).

8 21. Duan, H. et al. Chilling Prospect: Climate Change Effects of Mismanaged

9 Refrigerants in China. Environ. Sci. Technol. 52, 6350-6356 (2018).

10 22. Safeguarding the Ozone Layer and the Global Climate System: Issues Related to

11 Hydrofluorocarbons and Perfluorocarbons (SROC). (IPCC/TEAP, Cambridge University 12 Press, 2005).

13 23. Lunt, M. F. et al. Continued Emissions of the Ozone-Depleting Substance Carbon

14 Tetrachloride From Eastern Asia. Geophys. Res. Lett. (2018). doi:10.1029/2018GL079500

15 24. Miller, B. R. et al. Medusa: A Sample Preconcentration and GC / MS Detector

16 System for in Situ Measurements of Atmospheric Trace Halocarbons, Hydrocarbons, and

17 Sulfur Compounds. Anal. Chem. 80, 1536-1545 (2008).

18 25. Enomoto, T., Yokouchi, Y., Izumi, K. \& Inagaki, T. Development of an Analytical

19 Method for Atmospheric Halocarbons and Its Application to Airborne Observation. J. Jpn.

20 Soc. Atmospheric Environ. 40, 1-8 (2005).

21 26. Cullen, M. J. P. The Unified Forecast/Climate Model. Meteorol. Mag. 122, (1993).

22 27. Chipperfield, M. P. et al. Multimodel estimates of atmospheric lifetimes of long-lived

23 ozone-depleting substances: Present and future: Model Estimates of Atmospheric

24 Lifetimes. J. Geophys. Res. Atmospheres 119, 2555-2573 (2014). 
1 28. Ganesan, A. L. et al. Characterization of uncertainties in atmospheric trace gas

2 inversions using hierarchical Bayesian methods. Atmospheric Chem. Phys. 14, 3855-3864 3 (2014).

4 29. Green, P. J. Reversible jump Markov chain monte carlo computation and Bayesian 5 model determination. Biometrika 82, 711-732 (1995).

6 30. Stohl, A. et al. An analytical inversion method for determining regional and global 7 emissions of greenhouse gases : Sensitivity studies and application to halocarbons. $8 \quad$ Atmospheric Chem. Phys. 9, 1597-1620 (2009).

9 31. O’Doherty, S. et al. In situ chloroform measurements at Advanced Global 10 Atmospheric Gases Experiment atmospheric research stations from 1994 to 1998. J. 11 Geophys. Res. 106, 20429-20444 (2001).

12 32. Rigby, M. et al. Re-evaluation of the lifetimes of the major CFCs and $\mathrm{CH} 3 \mathrm{CCl} 3$ using atmospheric trends. Atmospheric Chem. Phys. 13, 2691-2702 (2013).

14 33. Rigby, M. et al. Recent and future trends in synthetic greenhouse gas radiative forcing. Geophys. Res. Lett. 41, 2623-2630 (2014).

17 Acknowledgements

We are indebted to the site operators who oversee the day-to-day running of AGAGE, NIES, and NOAA stations. We particularly thank the NASA Upper Atmosphere Research Program for its continuing support of AGAGE, including providing modelling, field station and instrumentation support though grant NNX16AC98G to MIT, and supporting the overall experimental programme through grants NNX16AC96G and NNX16AC97G to SIO.

Observations at Cape Grim are supported largely by the Australian Bureau of Meteorology,

24 CSIRO, the Australian Department of the Environment and Energy (DoEE) and Refrigerant

25 Reclaim Australia (RRA). Mace Head, Ireland, is supported by the Department for Business, 
1 Energy \& Industrial Strategy (BEIS, UK, formerly the Department of Energy and Climate

2 Change (DECC)) contract 1028/06/2015 to the University of Bristol and the UK

3 Meteorological Office. Ragged Point, Barbados is supported by the National Oceanic and

4 Atmospheric Administration (NOAA, USA), contract RA-133-R15-CN-0008 to the

5 University of Bristol. L.W., M.L. and R.T. were supported by Natural Environment Research

6 Council grants NE/I027282/1 and NE/M014851/1. A.G. is supported by NERC Independent

7 Research Fellowship NE/L010992/1. S.P., T.L., S.L., M.K.P., and K.R.K. were supported by

8 Basic Science Research Program through the National Research Foundation of Korea (NRF)

9 funded by the Ministry of Education (No. NRF-2016R1A2B2010663). Observations at

10 Hateruma were partly supported by the Ministry of the Environment of Japan. S.M., B.H.

11 and G.D. are indebted to C. Siso, D. Mondeel, J.D. Nance, F. Moore, J.W. Elkins, B. Vasel,

12 C. Schultz, R. Schnell, and J.H. Butler for discussions and assistance with the NOAA

13 measurements considered here, which were made possible in part with support from the

14 NOAA Climate Program Office's AC4 program.

\section{Author contributions}

S.P., T.S., G.S.D., P.J.F., B.D.H., C.M.H., J.K., K.R.K., P.B.K, T.L., S.L., S.A.M., J.M., S.O., M.K.P., P.K.S., P.S., R.F.W., Y.Y. and D.Y. contributed observational data. L.M.W., A.L.R., X.F. and S.H. carried out atmospheric model simulations and inverse analysis with support from R.L.T., M.F.L. and A.L.G., and under the supervision of M.R., A.J.M., S.R. and R.G.P. The authors M.R., S.P., T.S., L.M.W., A.L.R and X.F. made an equal contribution to the work. All authors wrote the manuscript.

\section{Additional information}

Reprints and permissions information is available at http://www.nature.com/reprints. 
1 Competing interests: The authors declare no competing interests.

2 Correspondence and requests for materials should be addressed to sparky@knu.ac.kr

\section{$4 \quad$ Figures captions}

5 Figure 1. Dry-air mole fraction observations for CFC-11 at Gosan, Jeju Island, Republic of

6 Korea $\left(33.3^{\circ} \mathrm{N}, 126.2^{\circ} \mathrm{E}\right)$ and Hateruma, Japan $\left(24.1^{\circ} \mathrm{N}, 123.8^{\circ} \mathrm{E}\right)$. The grey lines show the

7 mole fraction observations at Cape Grim, Tasmania, Australia $\left(40.7^{\circ} \mathrm{S}, 144.7^{\circ} \mathrm{E}\right)$ for

8 comparison.

9 Figure 2. CFC-11 emissions derived from atmospheric observations. The upper part of the

10 figure shows global emissions estimates derived using a global box model and AGAGE

11 (green diamonds) and NOAA (orange crosses) mole fraction observations of baseline air. The lower part of the figure shows emissions estimates derived for eastern mainland China using measurements from Gosan (AGAGE, Republic of Korea) and Hateruma (NIES, Japan) and

14 four inverse modelling frameworks: NAME-HB (yellow crosses), NAME-InTEM (blue squares), FLEXPART-MIT (pink circles) and FLEXPART-Empa (grey triangles). Inventorybased estimates for CFC-11 emissions from eastern mainland China (determined as total

17 Chinese emissions scaled by the fraction of the population, $35 \%$, that reside in that part of the domain) are shown as a solid black line and a projection of the inventory estimates are shown as a dashed line ${ }^{7}$. All lines and symbols represent the a posteriori mean, and the shading, the 1-standard deviation uncertainty. Global emissions are higher than in a previous study using NOAA data ${ }^{3}$, because a shorter atmospheric lifetime was used here ${ }^{20}$.

Figure 3. Spatial distribution of the derived CFC-11 fluxes in the NAME-HB inversion. a) mean emissions for 2008 - 2012; b) mean emissions 2014 - 2017; c) Change in emissions from 2008 - 2012 to 2014 - 2017. Equivalent plots for the other inversions are shown in Extended Data Figure 16. The hatched areas indicate regions of the domain to which the 
observations have low sensitivity (Extended Data Figure 2), and therefore, from which the

2 derived emissions have high uncertainty. As a result, only emission magnitudes and emission

3 changes for the non-hatched regions are included in the values quoted in the main text.

4

\section{Methods}

6 Data

7 Observations were made at a frequency of around 34 measurements per day at the remote

8 AGAGE stations using gas chromatograph (GC) electron capture detection ${ }^{4}$. These stations

9 are at Mace Head, Ireland (53.3 N, 9.9 W), Trinidad Head, CA, USA (41.1 N, 124.2 W),

10 Ragged Point, Barbados (13.2 N, 59.4 W), Cape Matatula, American Samoa (14.2 S, 170.7

11 W) and Cape Grim, Tasmania, Australia (40.7 S, 144.7 E). At the AGAGE station at Gosan,

12 Republic of Korea (33.3 N, 126.2 E) around 12 measurements were made each day using

13 GC-mass spectrometry (MS) ${ }^{24}$. Measurements at the NIES, AGAGE-affiliate station at

14 Hateruma, Japan (24.1 N, 123.8 E) were made at a frequency of around 20 measurements per 15 day using GC-MS ${ }^{25}$. AGAGE measurements are reported on the Scripps Institution of

16 Oceanography (SIO) 2005 scale and NIES measurements are reported on the NIES-08 scale.

17 Hateruma data were converted to the SIO-05 scale by multiplying by an intercalibration

18 factor of 0.996 , determined through exchange of air samples. The estimated repeatability of

19 the Hateruma data was approximately $0.8 \%$, and the average repeatability of the Gosan data

20 was approximately $0.2 \%$, although this varies throughout the time-series.

21

22 NOAA's background atmospheric CFC-11 measurements from three different methods were

23 combined into a single atmospheric history for this analysis (for the combined record, see

24 ftp://ftp.cmdl.noaa.gov/hats/cfcs/cfc11/combined/ for data and

25 https://www.esrl.noaa.gov/gmd/hats/combined/CFC11.html for a description of the 
1 methodology). Those methods include GC-ECD measurements at hourly frequency using on-

2 site instrumentation, and the GC-ECD and GC-MS (the latter included only since 2010)

3 analysis of glass and stainless-steel flasks collected approximately weekly at 12 locations

4 across the globe. Flasks were analysed in Boulder, CO, USA ${ }^{3}$. NOAA data are reported

5 using the NOAA-2016 scale.

6

7 Differences between the NOAA-2016 and the SIO-05 scales are $1 \%$ or less ${ }^{4}$. Therefore,

8 differences between the AGAGE and NOAA-derived global emissions (Figure 5) are not

9 strongly influenced by calibration differences. Global inversions were carried out using

10 AGAGE and NOAA data on their respective calibration scales. Uncertainties due

11 inaccuracies in the calibration scales were not included in the global emissions estimates (see

12 below).

14 Regional atmospheric chemical transport models

15 We use two backward-running Lagrangian chemical transport models to estimate the 16 sensitivity of the atmospheric observations to fluxes in the eastern Asian region: NAME ${ }^{14}$

17 and FLEXPART ${ }^{15}$. Both models simulate transport of CFC-11 by advection and random

18 diffusion, by atmospheric turbulence, of hypothetical particles of the gas. The "footprint" of

19 each measurement, calculated from these simulations, describes the sensitivity of the

20 observation to emissions from surrounding model grid cells (Extended Data Figure 11). A

21 comparison of the simulated and observed data is presented in Extended Data Figure 12.

23 NAME was driven by meteorological fields from the UK Met Office Unified Model ${ }^{26}$, the

24 spatial resolution of which increased in the study period from 0.563 to $0.141^{\circ}$ longitude and

250.375 to $0.094^{\circ}$ latitude, whilst the temporal resolution has remained fixed at 3 hours. 20,000 
1 particles per hour were transported backward in time for 30 days in NAME in a domain

2 bounded at $5^{\circ} \mathrm{S}$ and $74^{\circ} \mathrm{N}$ and $55^{\circ} \mathrm{E}$ and $192^{\circ} \mathrm{E}$. When particles are within the lowest $40 \mathrm{~m}$ of

3 the atmosphere, it was assumed that they interacted with the surface and could intercept

4 emissions sources $^{17,23}$. The NAME-HB framework uses a climatological a priori estimate of

5 the mole fraction at the boundary of the domain from the NASA 3D GEOS Chemistry

6 Climate $\mathrm{Model}^{27}$. In contrast, the NAME-InTEM inversion estimated the baseline mole

7 fraction at each measurement station (see below).

8

9 FLEXPART was run in different modes by the MIT and Empa groups. FLEXPART-Empa

10 released 50,000 particles in each 3-hour interval and traced them backwards in time for 10

11 days, driven by operational ECMWF analysis with $1^{\circ} \times 1^{\circ}$ global resolution and $0.2^{\circ} \times 0.2^{\circ}$

12 resolution for north-eastern China $\left(105^{\circ} \mathrm{E}\right.$ to $125^{\circ} \mathrm{E}$ and $30^{\circ} \mathrm{N}$ to $\left.50^{\circ} \mathrm{N}\right)$. FLEXPART-MIT

13 released 40,000 particles in each 3-hour interval and traced them backwards in time for 20

14 days in a global domain, driven by operational ECMWF analysis at $1^{\circ} \times 1^{\circ}$ global resolution.

16 Regional inversion frameworks

17 Fluxes were derived using four different Bayesian inversion frameworks. Bayesian inverse modelling systems use an a priori estimate of emissions, which, when combined with

19 chemical transport models, can be subsequently updated to bring the models into consistency

20 with observations. In our study, different a priori estimates were used in each regional

21 inversion, although in every case these estimates primarily served to weakly limit the spatial area from which emissions were inferred (they were limited to being over land, but not

23 further constrained to any particular land-based regions), and to provide the inversion with an

24 approximate magnitude for the fluxes (by typically being some fraction of the global total).

25 The magnitude of the a priori emissions was constant for each year of the study, so that any 
1 temporal variation in the a posteriori emissions was driven only by the data. Bayesian

2 frameworks require that some uncertainty is placed on these prior estimates along with an

3 estimate of the model-measurement uncertainty covariance (see below). The hierarchical

4 approach allows these uncertainty factors, along with other parameters, to be estimated in the

5 inversion ${ }^{28}$. Our inversions were shown to be relatively insensitive to the choice of prior

6 emissions magnitude or distribution (Extended Data Figure 14).

7

8 The trans-dimensional hierarchical Bayesian inversion used with NAME footprints (NAME-

$9 \mathrm{HB})$ estimates the model-measurement uncertainty in the inversion ${ }^{28}$. Furthermore, the level

10 of spatial disaggregation of the emissions field can also be explored ${ }^{16}$. This scheme is solved

11 using reversible-jump Markov chain Monte Carlo (rj-MCMC) ${ }^{29}$. The inversion is split into an

12 inner domain $\left(74^{\circ} \mathrm{W}\right.$ to $160^{\circ} \mathrm{W}, 22^{\circ} \mathrm{N}$ to $\left.50^{\circ} \mathrm{N}\right)$, where spatial disaggregation can occur, and

13 an outer domain split into four fixed regions to the North, South, East and West extending to

14 the boundary of the NAME domain. Emissions are estimated at variable resolution

15 (determined by the rj-MCMC algorithm) in the inner domain and from the four fixed regions

16 outside this domain. Simultaneously, boundary conditions at the four domain edges were

17 solved for by scaling the a priori mole fraction fields at these edges. Our a priori estimate of

18 CFC-11 emissions were $20 \mathrm{Gg} \mathrm{yr}^{-1}$ distributed uniformly over land (not water) in the inner

19 domain and a further $20 \mathrm{Gg} \mathrm{yr}^{-1}$ in the outer domain, meaning that the combined domain

20 accounts for roughly two thirds of global emissions. This results in national a priori

21 emissions estimates of $17.3 \mathrm{Gg} \mathrm{yr}^{-1}$ for China (5.5 $\mathrm{Gg} \mathrm{yr}^{-1}$ for eastern mainland China) and

$221.2,0.31$ and $0.42 \mathrm{Gg} \mathrm{yr}^{-1}$ for Japan, Republic of Korea and D. P. R. Korea, respectively.

23 Within each country, these prior fluxes were uniformly distributed in space. The uncertainty

24 on these flux estimates was assumed to be lognormally distributed, with a mode fixed to the $a$

25 priori estimated emission and a logarithmic standard deviation of 10 , defined as the square- 
1 root of the ratio of the exponential of the mean to the mode. Model uncertainties are informed

2 by the data in the hierarchical framework. The model error was assumed to be normally

3 distributed with zero mean and a standard deviation that was allowed to vary, with equal

4 probability, between 1 and 20 ppt, with an autocorrelation timescale between 1 and 120

5 hours. The measurement data and footprints were averaged into daily values, with a fixed

6 measurement uncertainty equal to the repeatability of the raw measurements plus the

7 variability of the measurements within the daily averaging period (a measure of the sub-daily

8 fluctuations that would not be resolved by the inversion). Emissions were assumed constant

9 throughout the year, and emissions and monthly boundary condition scaling factors were

10 estimated independently within each year.

12 Output from NAME was also used with the InTEM inversion framework (NAME-InTEM) ${ }^{17}$.

13 A priori CFC-11 estimates were given large uncertainties, namely 22 (0-147) $\mathrm{Gg} \mathrm{yr}^{-1}$ for

14 China, 5 (0-35) Gg $\mathrm{yr}^{-1}$ for eastern mainland China, and $2(0-20), 0.3(0-3.2)$ and $0.4(0-3.8)$

$15 \mathrm{Gg} \mathrm{yr}^{-1}$ for Japan, Republic of Korea and D. P. R. Korea, respectively. This is based on 50

$16 \mathrm{Gg} \mathrm{yr}^{-1}$ uniformly emitted across the whole eastern Asian land area. Observational

17 uncertainty was time varying and was estimated as the variability of the observations in a six-

18 hour moving window plus the measurement repeatability determined from repeat

19 measurements of the on-site calibration standards. Model uncertainty was estimated every

20 two hours as the larger of the median of all pollution events at each station in a year or $16.5 \%$

21 of the magnitude of the pollution event. A temporal correlation of 12 hours was assumed in

22 the model uncertainty at each station. An analytical solution was found that minimized the

23 residual between the model and the observations and the difference between the posterior and

24 prior flux estimate, balanced by the uncertainties of both. The mole fraction baseline at each

25 station was estimated in the inversion following Arnold et al. (2018) ${ }^{17}$. The variable 
1 resolution of the inversion grid was calculated and refined within InTEM based on the

2 magnitude of the footprint and emissions from each grid box. The inversions were run

3 multiple (24) times per year, each time with a randomly generated sub-sample (90\%) of the

4 available observations (10\% removed in 5-day blocks), to further explore the uncertainty.

5 Emissions and uncertainties were averaged across the 24 individual inversions thereby

6 assuming $100 \%$ correlation between uncertainties in these separate inversions.

8 The FLEXPART-MIT inversion derived fluxes using FLEXPART with an analytical

9 Bayesian framework ${ }^{30}$. Prior flux fields were set to be spatially uniform over continental

10 eastern Asia. Emissions were solved within variable-resolution grid cells with the finest grid

$11\left(1^{\circ} \times 1^{\circ}\right)$ in eastern China and other eastern Asian countries ${ }^{31}$. A priori estimates of CFC-11

12 flux were 14.5 $\mathrm{Gg} \mathrm{yr}^{-1}$ for China (1.5 $\mathrm{Gg} \mathrm{yr}^{-1}$ for eastern mainland China) and 1.2, 0.27 and

$130.27 \mathrm{Gg} \mathrm{yr}^{-1}$ for Japan, Republic of Korea and D. P. R. Korea, respectively. The uncertainty

14 on each grid cell was arbitrarily set to $1000 \%$ and the spatial correlation length was assumed

$15300 \mathrm{~km}$, so that the total a priori emission uncertainty for China, for example, was $43.2 \mathrm{Gg}$

$16 \mathrm{yr}^{-1}$. Baseline mole fractions were estimated at each site every 7 days $^{31}$. Model-measurement

17 uncertainty for each 24-hour-averaged observation was estimated as the quadratic sum of $1 \%$

18 of the baseline value (as a measure of baseline uncertainty), the measurement repeatability

19 and the standard deviation of the 24-hourly variability (as a measure of the model-data

20 "mismatch" uncertainty). Similarly to InTEM, a posteriori fluxes were derived using an

21 analytical approach that minimises a cost function that weights the measurement-model

22 residuals and the a priori estimate by their respective uncertainties.

24 Fluxes were derived with a second FLEXPART-based approach but using the FLEXPART-

25 Empa simulations and an alternative analytical Bayesian framework ${ }^{19}$. Prior fluxes were set 
1 to a homogeneous value over all land areas with total Chinese and eastern mainland Chinese

2 emissions of $14.9 \mathrm{Gg} \mathrm{yr}^{-1}$ and $2.7 \mathrm{Gg} \mathrm{yr}^{-1}$, respectively, (0.66, 0.18, $0.22 \mathrm{Gg} \mathrm{yr}^{-1}$ for Japan,

3 Republic of Korea and D. P. R. Korea). Observations were aggregated to 3-hourly averages

4 to s gridded annual mean emissions. The baseline was optimized as part of the state vector,

5 independently for both sites. No a priori covariance between emissions and baseline were

6 assigned. Parameters describing the covariance structure of the data-mismatch uncertainty

7 matrix and the a priori uncertainty matrix were estimated using a maximum likelihood

8 approach $^{19}$. These parameters include an estimate of the model uncertainty at the individual

9 sites, the a priori baseline uncertainty and its temporal correlation length scale, a domain-

10 total a priori uncertainty, and a spatial correlation length scale. The domain-total a priori

11 uncertainty was estimated to range from $\pm 45 \%$ (in 2008) to $\pm 125 \%$ (in 2016), which

12 translates to a regional a priori uncertainty of $\pm 100 \%$ to $\pm 270 \%$ for the eastern mainland

13 China region.

15 Where the emissions, derived from multiple regional inversions and multiple years, were combined, we took the mean of the $a$ posteriori annual inversion means. Mean emissions are

17 calculated throughout the text for the periods 2008 - 2012 and $2014-2017$ (inclusive). The year 2008 was used to bound the first period, when a whole year of Gosan data first became available. A previous study ${ }^{3}$, which estimated global emissions using NOAA data, omitted the year 2013, because global emissions in this year appeared to be intermediate between two periods of more constant emission. Whilst our estimates, which use multiple models and measurement networks, are less clear on whether such an intermediate period occurred, we

23 also omit the year 2013, so that our estimates can be more directly compared with this

24 previous work. Uncertainties in the multi-inversion means were calculated from the $a$ posteriori uncertainty from the individual models and years, assuming that these uncertainties 
1 were fully correlated (i.e. assuming that the different inversions did not provide statistically

2 independent estimates of emissions). The latter assumption attempts to account for the fact

3 that the inversions share the same models, which will suffer from similar systematic

4 uncertainties (in the case of the two NAME inversions or two FLEXPART inversions). Such

5 systematic uncertainties are not explored in any of the inversion frameworks and are

6 therefore not reflected in the individual a posteriori emission estimates. Therefore, the

7 individual uncertainty estimates are likely to be underestimates of the full uncertainty (as

8 seen by the non-overlapping uncertainties in some years in, for example, Figure 5). It is also

9 possible that, for the same reason, our multi-inversion uncertainty is an under-estimate.

11 Based on a consideration of the sensitivity of the observations to potential sources

12 surrounding the measurement sites (note that the NOAA and AGAGE network currently have

13 a limited number of stations which are close enough to industrialised regions for the detection

14 of regional emission sources), we have opted to focus on the total emissions rise from a

15 region that we denote eastern mainland China (Extended Data Figure 2, a similar sub-

16 division was also used for western Japan). Further away from this region, atmospheric mixing

17 of plumes that emanate from emissions sources makes their impact on the observations small,

18 compared to measurement and modelling uncertainty (which is why the existing, sparse

19 network is insensitive to large parts of the globe). The choice of which Chinese provinces to

20 include in this region was somewhat subjective and could alter the derived emissions rise and

21 its uncertainty. Inclusion of the provinces adjacent to our eastern mainland China region

22 (excluding the very large Inner Mongolia) would lead to an increase of approximately $15 \%$ in

23 the emissions rise derived between 2008-2012 and 2014-2017. However, the associated

24 uncertainty increased by around 30\%. We note that the use of a larger region would explain a 
1 larger (but more uncertain) fraction of the global emissions rise than obtained from the

2 eastern mainland China region alone.

\section{Global average emissions estimation}

5 Global emissions were estimated from AGAGE or NOAA data with a least-squares method

6 used previously for global CFC-11 emissions estimation ${ }^{3}$. Monthly mean baseline-filtered

7 observations ${ }^{32}$ from the five remote AGAGE stations or the NOAA flask and in situ sites

8 were averaged into semi-hemispheres, separated at the equator and $30^{\circ}$ north or south

9 (NOAA data were weighted by the sine of their latitude within each semi-hemisphere). Mole

10 fractions were simulated for each semi-hemisphere using a 12-box model of the atmosphere

11 with vertical divisions at $500 \mathrm{hPa}$ and $200 \mathrm{hPa}^{33}$. Seasonally varying, inter-annually repeating

12 advection and diffusion parameters were used to transport CFC-11 between model boxes.

13 Loss was assumed only to occur in the stratospheric boxes, at a rate that was adjusted to

14 provide a global mean steady-state lifetime of 52 years ${ }^{20}$. Uncertainties related to the model's 15 ability to simulate the data consisted of the sum of two factors. The first term, equal to the

16 baseline variability in each month determined by the AGAGE network, was assumed to be a

17 measure of the temporal representation error between the model and the data. A second fixed

18 uncertainty $(0.3 \%)$, equal to the approximate level of disagreement between the AGAGE and

19 NOAA monthly semi-hemispheric means, was used to parameterize spatial and temporal

20 differences in the sampling between the networks and uncertainties in the propagation of

21 calibration scales to the sites. These uncertainties are propagated through to the emissions

22 estimates. However, our estimates do not include an uncertainty contribution due to

23 calibration scale uncertainty or atmospheric lifetime uncertainty ${ }^{33}$. These terms are important

24 for quantifying the absolute derived emissions values but have little influence on the change

25 in emissions between time periods, which is the primary focus of this paper. Similarly to 
1 previous studies ${ }^{3,4}$, our uncertainties do not include a contribution due to the assumption of

2 inter-annually repeating dynamics. To estimate the implications of this assumption would

3 require three-dimensional global model simulations, which were not available for this work.

\section{Data Availability}

6 Data from remote AGAGE stations and Gosan data are available from the AGAGE website

7 (agage.mit.edu). Hateruma data are available at World Data Center for Greenhouse Gases

8 (http://ds.data.jma.go.jp/gmd/wdcgg/wdegg.html). NOAA data are taken as the "combined

9 set" data record (a best-estimate record based on flask samples and in-situ measurements),

10 available from the NOAA Global Monitoring Division data server

11 (https://www.esrl.noaa.gov/gmd/dv/ftpdata.html, or more specifically

12 ftp://ftp.cmdl.noaa.gov/hats/cfcs/cfc11/combined/).

14 Code availability:

15 The inversion models of NAME-HB, NAME-InTEM, FLEXPART-MIT and FLEXPART-

16 Empa are used to determine the regional CFC-11 emissions. Enquiries about the model codes

17 should be directed to M.R., A.J.M., S.R. and R.G.P. Licences to use NAME and InTEM are available for research purposes via a request to the UK Met Office or on request from A.J.M. and A.L.R... The code for the NAME-based hierarchical Bayesian inversion (NAME-HB) is available on request from M.R. and L.M.W.. The code of the dispersion model FLEXPART is available from www.flexpart.eu. The code for the FLEXPART-based Bayesian inversion (FLEXPART-MIT) is available on request from X.F.. The inversion code used by Empa is available from https://doi.org/10.5281/zenodo.1194642 or on request from S.H.. 
3 Extended Data Figure 1. Box and whisker plots of mole fractions measured at different

4 sites in the AGAGE network and at affiliated AGAGE sites. Boxes indicate the $25^{\text {th }}$ and

$575^{\text {th }}$ percentiles of the individual measurement data (approximately 2-hourly), with the

6 median shown as a line within each box. The whiskers show the $10^{\text {th }}$ and $90^{\text {th }}$ percentiles. The

7 lower percentiles are typically representative of baseline ("unpolluted") mole fractions, and

8 the difference between the lower and higher percentiles indicates the magnitude of the above-

9 baseline events due to the interception of air masses containing recently emitted CFC-11.

10 Extended Data Figure 2. Mean sensitivity of observations at Gosan (33.3 N, 126.2 E)

11 and Hateruma (24.1 N, 123.8 E) to emissions from 2008 to 2017 from the NAME model

12 simulation. The black triangles indicate two stations. Thin grey lines show the boundaries of

13 the provinces within the region we denote "eastern mainland China". This region contains the

14 provinces Anhui, Beijing, Hebei, Jiangsu, Liaoning, Shandong, Shanghai, Tianjin and

15 Zhejiang. The west of the grey line transecting Japan, containing the regions Chūgoku,

16 Kansai, Kyūshū \& Okinawa, and Shikoku, is the region we denote "western Japan".

17 Extended Data Figure 3. A comparison of simulated and observed mole fractions at

18 Gosan and Hateruma from the four inversion frameworks (left hand panel). Residuals

19 between the simulated and observed mole fractions (data minus model; right hand panel).

20 One standard deviation model-data mismatch uncertainties assumed in the inversions are

21 indicated by shading. Simulated mole fractions are derived from the a posteriori emissions.

22 For the NAME-InTEM, FLEXPART-EMPA, NAME-HB and FLEXPART-MIT inversions,

23 2-hourly, 3-hourly, 24-hourly and 24-hourly averaging was applied to the model and data,

24 respectively. 
1 Extended Data Figure 4. Inverse emissions estimates for eastern mainland China

2 derived using different data sets: only the Gosan CFC-11 measurements (green dashed line

3 with crosses), only the Hateruma (red dotted line with diamonds) CFC-11 measurements, or

4 both measurement records in the inversion analysis (pink solid line with circles). The

5 estimates shown use the (a) NAME-HB, (b) NAME-InTEM, (c) FLEXPART-MIT and (d)

6 FLEXPART-Empa inversion techniques.

7 Extended Data Figure 5. Derived emissions for eastern mainland China for different $a$

8 priori emission magnitudes and different spatial distributions. The inversions are as

9 described in Methods ("a priori" - pink solid with circles), with a priori emissions twice as

high (" $2 \mathrm{x}$ a priori" - green dashed line with crosses), or the same magnitude but distributed in

11 space according to population density ("population weighted" - red dotted line with

12 diamonds). Figures show the estimates using the (a) NAME-HB, (b) NAME-InTEM, (c)

13 FLEXPART-MIT and (d) FLEXPART-Empa inversion methods.

14 Extended Data Figure 6. Top-down emissions estimates for (a) western Japan, (b) the

15 Republic of Korea and (c) the Democratic People's Republic of Korea using the NAME-

16 HB (yellow exes), NAME-InTEM (blue squares), FLEXPART-MIT (pink circles) and

17 FLEXPART-Empa (grey inverted triangles) inverse frameworks described in Methods. See

18 Extended Data Figure 2 for the definition of the western Japan region. All lines and symbols

19 are the a posteriori mean and the shading shows the one standard deviation uncertainty.

20 Extended Data Figure 7. Mean CFC-11 emission fluxes and difference maps, similar to

21 Figure 3, for NAME-InTEM (a-c), FLEXPART-MIT (d-f) and FLEXPART-Empa (g-i) using the inversion framework described in the Methods. Figures (a), (d) and (g) show the average spatial emissions for the period 2008-2012; (b), (e) and (h) show the average spatial emissions for the period 2014-2017; and (c), (f) and (i) show the difference in emissions from 
2 FLEXPART-Empa respectively.

3 Extended Data Figure 8. The implied bank release fraction of CFC-11 from the eastern

4 mainland China region defined in Extended Data Figure 2, if there is no new production.

5 This calculation assumes that the 2008 emissions estimate are $5 \%$ of the bank size (estimated

6 to be toward the upper limit of expected bank fractional release rate ${ }^{3}$ ). The coloured lines

7 show the implied bank release fraction for each year after 2008, required to sustain the mean

8 a posteriori emissions from the NAME-HB (yellow), NAME-InTEM (blue), FLEXPART-

9 MIT (pink) and FLEXPART-Empa (grey) inversions. The black line shows the bank release

10 fraction from a bottom-up study for the whole of China ${ }^{7}$.

11 Extended Data Figure 9. Box and whisker plot of CFC-12 mole fraction at the Gosan measurement site. Boxes indicate the $25^{\text {th }}$ and $75^{\text {th }}$ percentiles of the (approximately 2 -

13 hourly) measurement data in a given year, and the median mole fraction is shown by the line 14 inside each box. The whiskers show the $10^{\text {th }}$ and $90^{\text {th }}$ percentiles. The lower percentiles are

15 typically representative of baseline mole fractions, and the difference between the lower and 16 higher percentiles indicates the magnitude of the above-baseline events due to the 17 interception of air masses containing recently emitted CFC-12. 


\section{Figures}

2

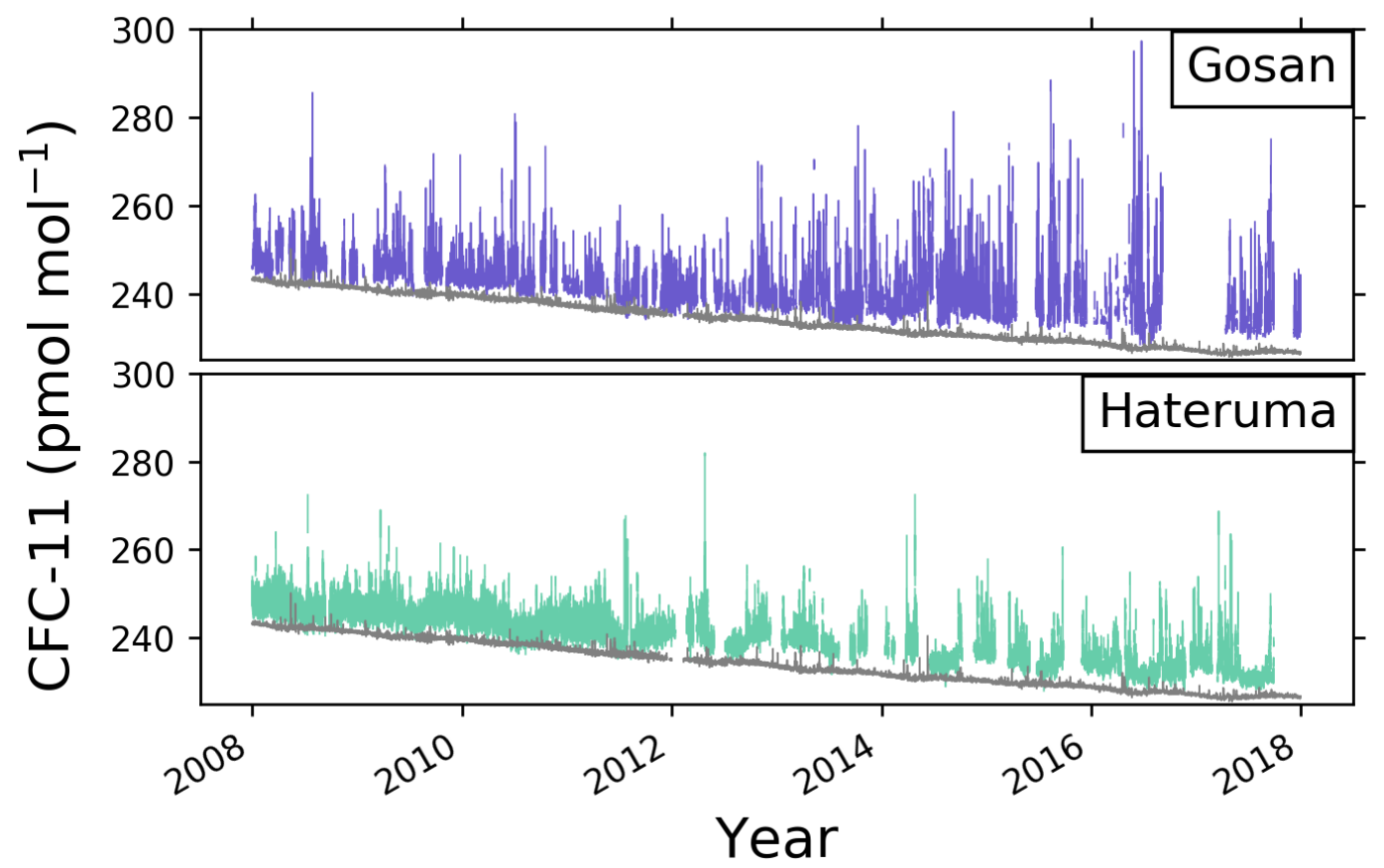

3

4 Figure 4. Dry-air mole fraction observations for CFC-11 at Gosan, Jeju Island, Republic of

5 Korea $\left(33.3^{\circ} \mathrm{N}, 126.2^{\circ} \mathrm{E}\right)$ and Hateruma, Japan $\left(24.1^{\circ} \mathrm{N}, 123.8^{\circ} \mathrm{E}\right)$. The grey lines show the

6 mole fraction observations at Cape Grim, Tasmania, Australia $\left(40.7^{\circ} \mathrm{S}, 144.7^{\circ} \mathrm{E}\right)$ for

7 comparison. 


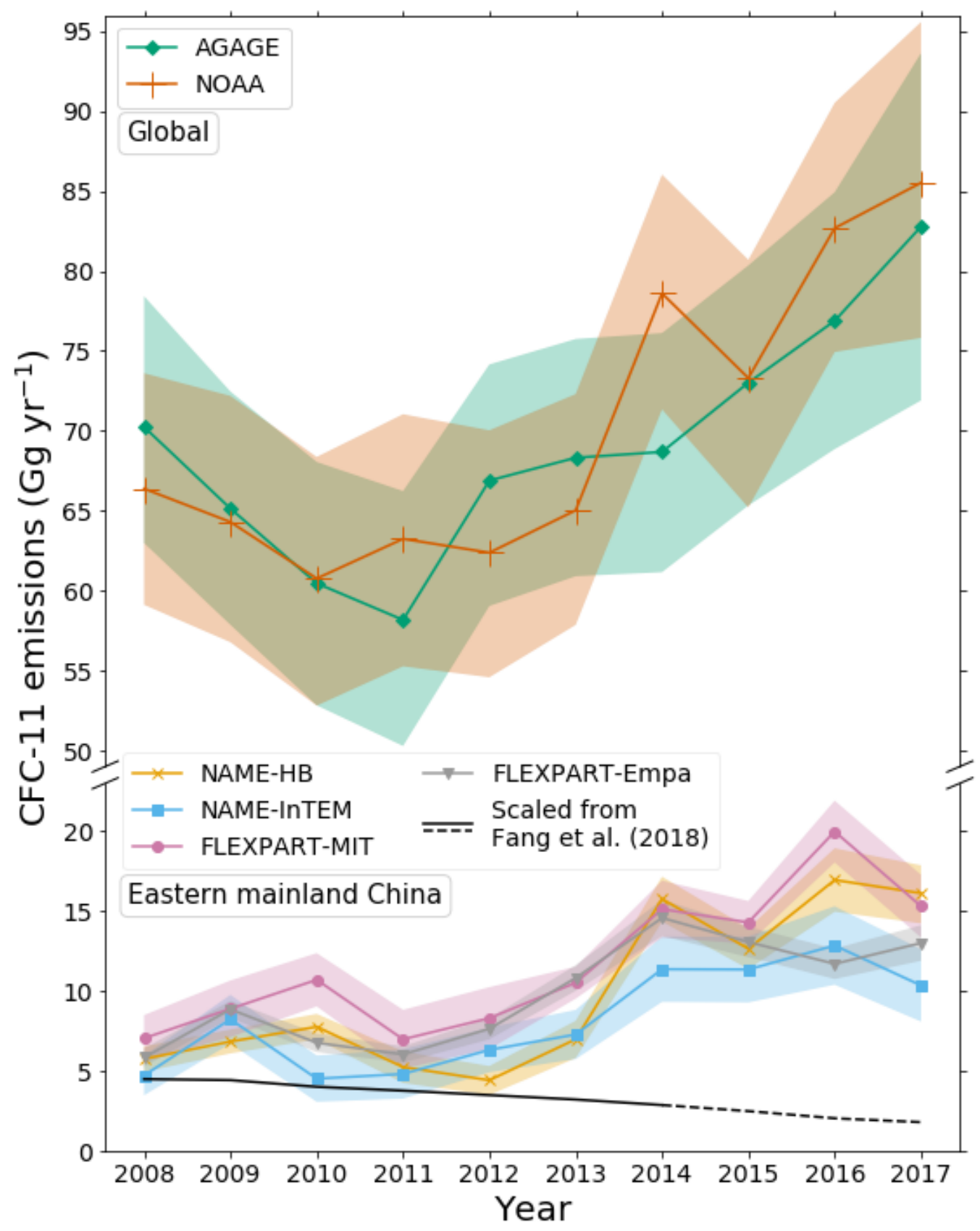

1

2 Figure 5. CFC-11 emissions derived from atmospheric observations. The upper part of the

3 figure shows global emissions estimates derived using a global box model and AGAGE

4 (green diamonds) and NOAA (orange crosses) mole fraction observations of baseline air. The

5 lower part of the figure shows emissions estimates derived for eastern mainland China using

6 measurements from Gosan (AGAGE, Republic of Korea) and Hateruma (NIES, Japan) and

7 four inverse modelling frameworks: NAME-HB (yellow crosses), NAME-InTEM (blue 
1 squares), FLEXPART-MIT (pink circles) and FLEXPART-Empa (grey triangles). Inventory-

2 based estimates for CFC-11 emissions from eastern mainland China (determined as total

3 Chinese emissions scaled by the fraction of the population, $35 \%$, that reside in that part of

4 the domain) are shown as a solid black line and a projection of the inventory estimates are

5 shown as a dashed line ${ }^{7}$. All lines and symbols represent the $a$ posteriori mean, and the

6 shading, the 1-standard deviation uncertainty. Global emissions are higher than in a previous

7 study using NOAA data ${ }^{3}$, because a shorter atmospheric lifetime was used here ${ }^{20}$. 

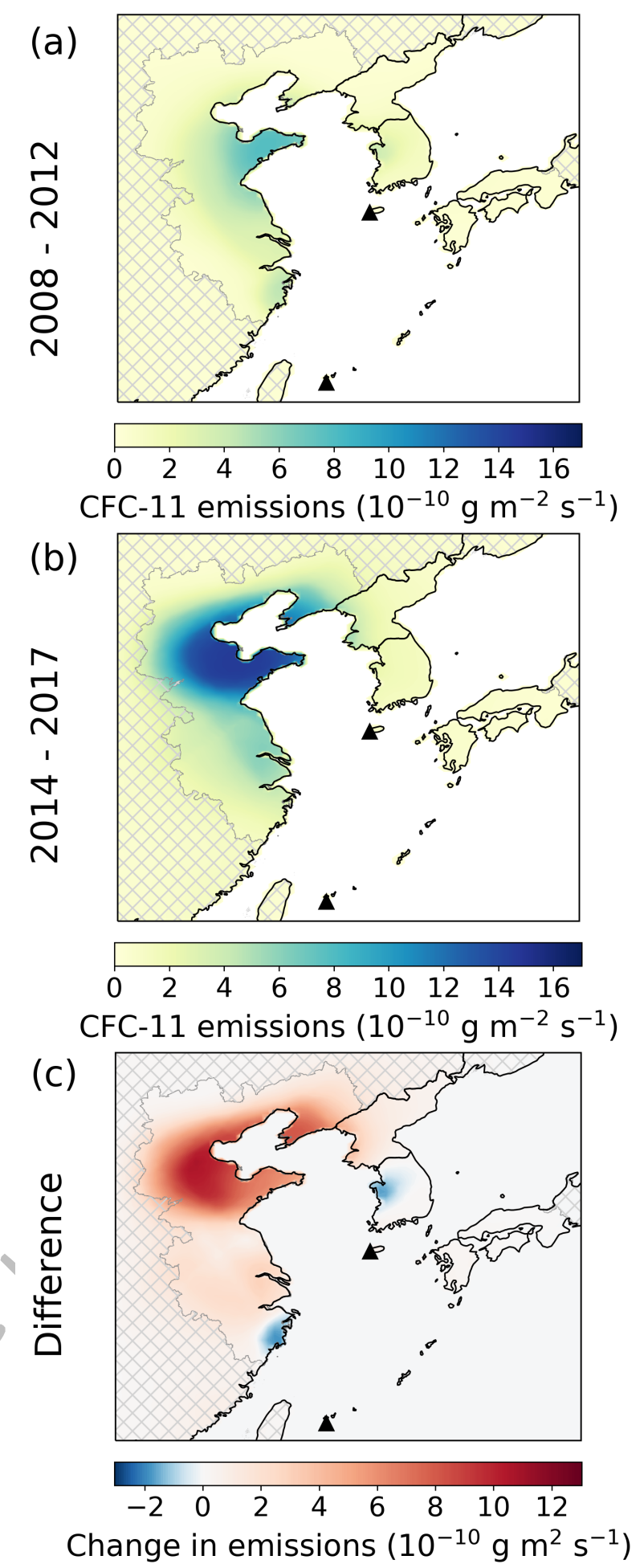

2 Figure 6. Spatial distribution of the derived CFC-11 fluxes in the NAME-HB inversion. a)

3 mean emissions for 2008 - 2012; b) mean emissions 2014 - 2017; c) Change in emissions

4 from 2008 - 2012 to 2014 - 2017. Equivalent plots for the other inversions are shown in

5 Extended Data Figure 16. The hatched areas indicate regions of the domain to which the 
1 observations have low sensitivity (Extended Data Figure 2), and therefore, from which the

2 derived emissions have high uncertainty. As a result, only emission magnitudes and emission

3 changes for the non-hatched regions are included in the values quoted in the main text.

4

5 


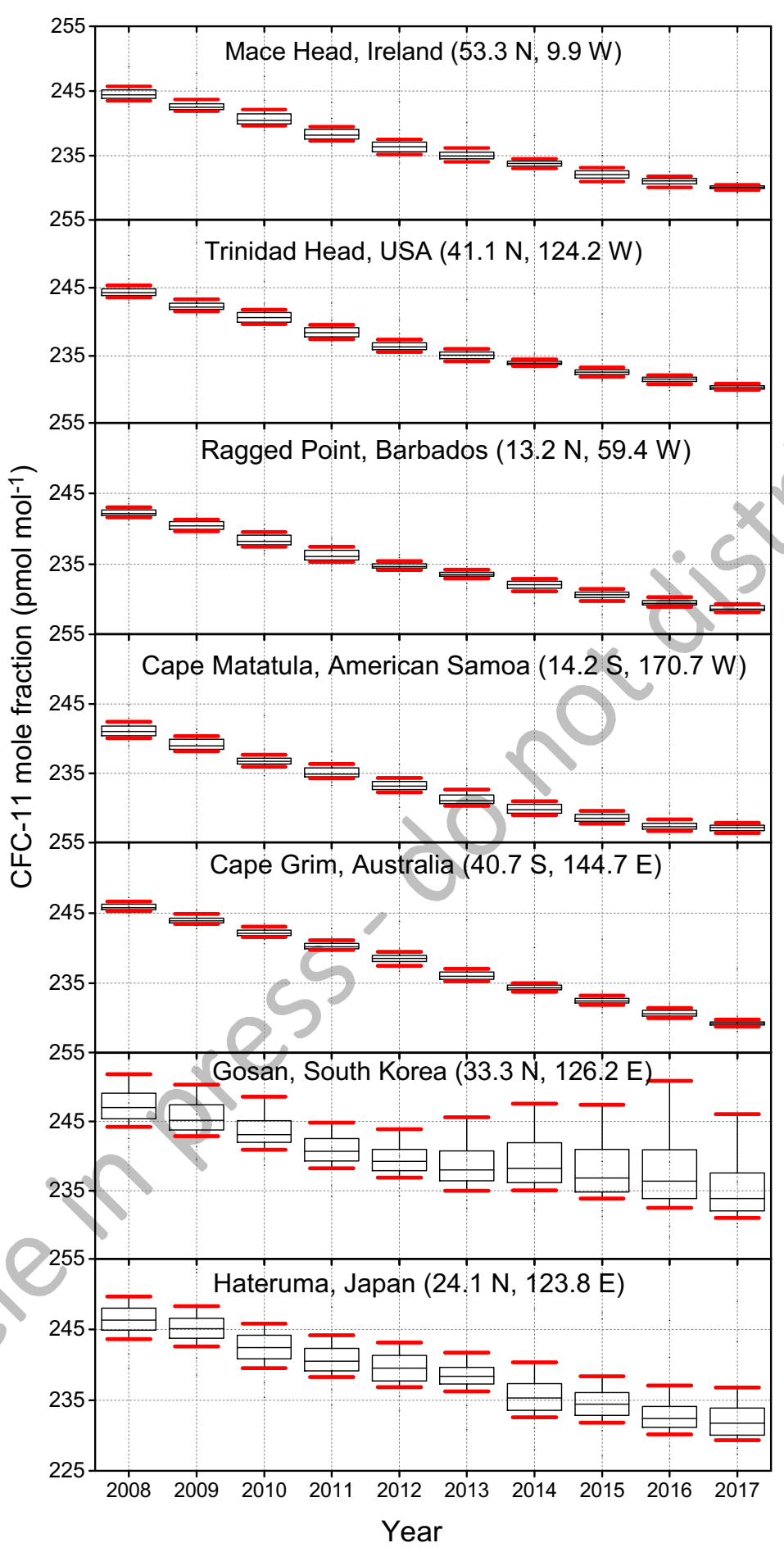

2

3 Extended Data Figure 10. Box and whisker plots of mole fractions measured at different sites

4 in the AGAGE network and at affiliated AGAGE sites. Boxes indicate the $25^{\text {th }}$ and $75^{\text {th }}$

5 percentiles of the individual measurement data (approximately 2-hourly), with the median 
1 shown as a line within each box. The whiskers show the $10^{\text {th }}$ and $90^{\text {th }}$ percentiles. The lower

2 percentiles are typically representative of baseline ("unpolluted") mole fractions, and the

3 difference between the lower and higher percentiles indicates the magnitude of the above-

4 baseline events due to the interception of air masses containing recently emitted CFC-11.
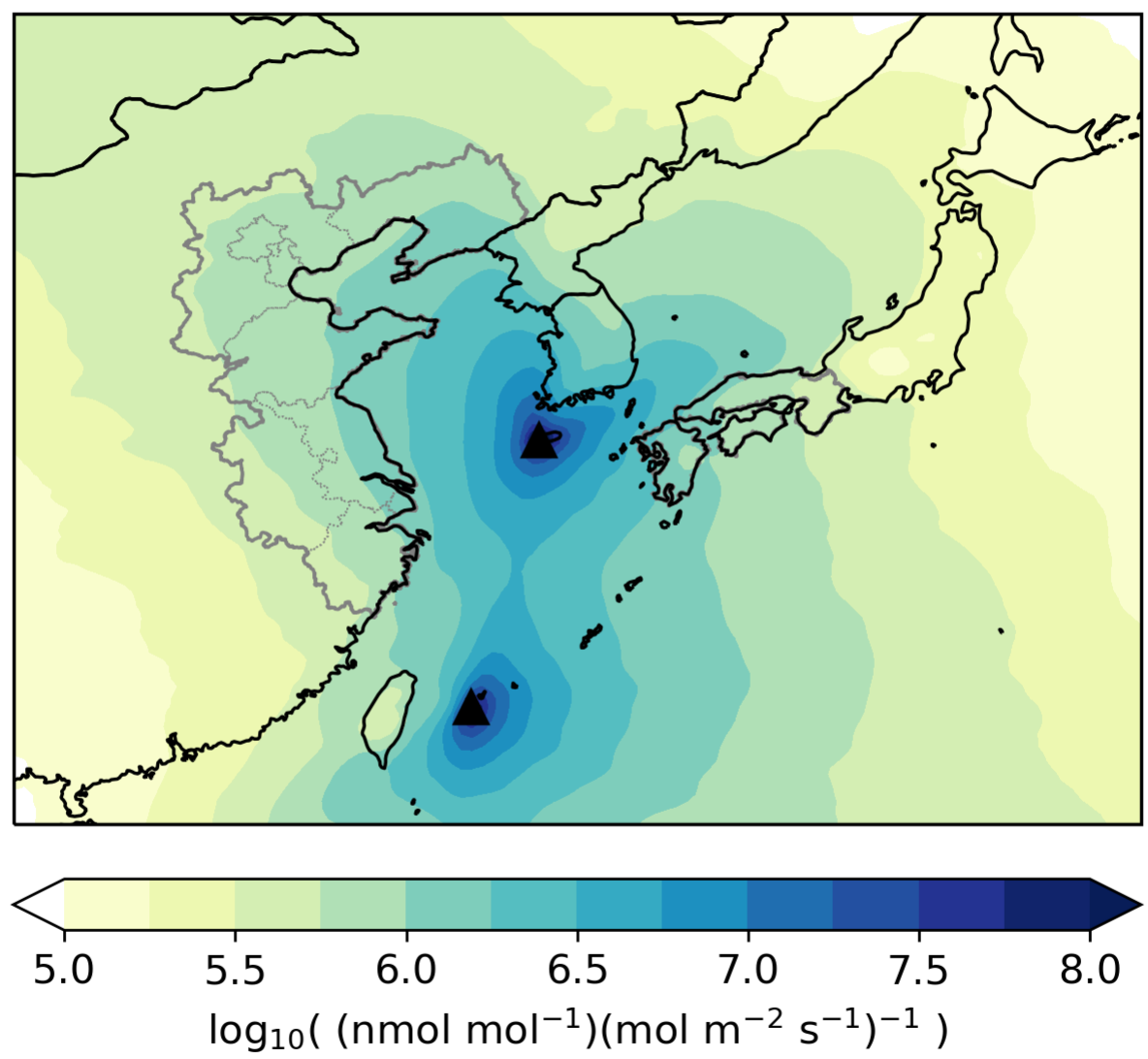

6

7 Extended Data Figure 11. Mean sensitivity of observations at Gosan (33.3 N, 126.2 E) and

8 Hateruma (24.1 N, 123.8 E), indicated by the black triangles, to emissions from 2008 to 2017

9 from the NAME model simulation. Thin grey lines show the boundaries of the provinces

10 within the region we denote "eastern mainland China". This region contains the provinces 
1 Anhui, Beijing, Hebei, Jiangsu, Liaoning, Shandong, Shanghai, Tianjin and Zhejiang. The

2 west of the grey line transecting Japan, containing the regions Chūgoku, Kansai, Kyūshū \&

3 Okinawa, and Shikoku, is the region we denote "western Japan". 


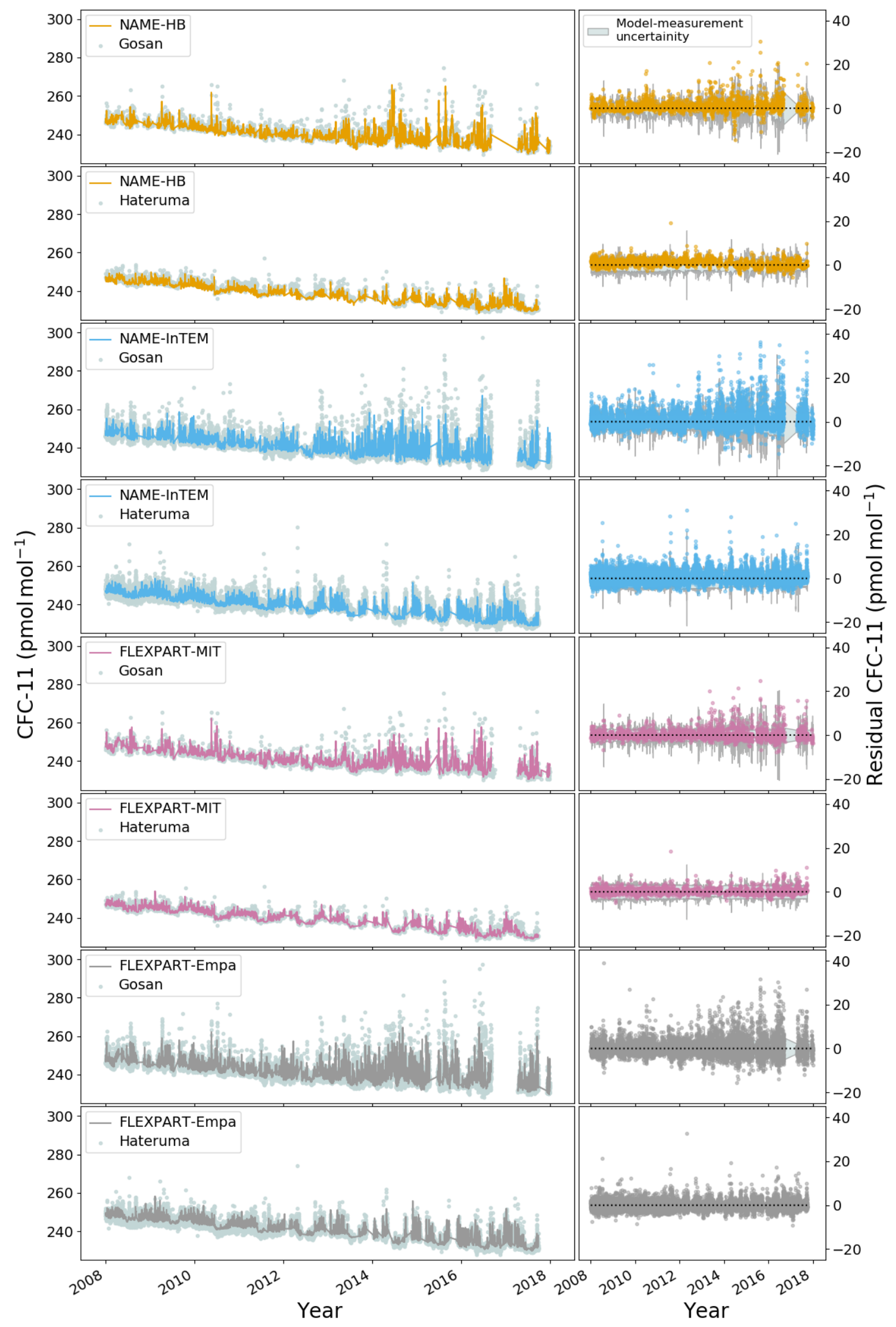

1

2 Extended Data Figure 12. Left hand panel: A comparison of simulated and observed mole

3 fractions at Gosan and Hateruma from the four inversion frameworks. Right hand panel: 
1 Residuals between the simulated and observed mole fractions (data minus model). One

2 standard deviation model-data mismatch uncertainties assumed in the inversions are indicated

3 by shading. Simulated mole fractions are derived from the a posteriori emissions. For the

4 NAME-InTEM, FLEXPART-EMPA, NAME-HB and FLEXPART-MIT inversions, 2-

5 hourly, 3-hourly, 24-hourly and 24-hourly averaging was applied to the model and data,

6 respectively.

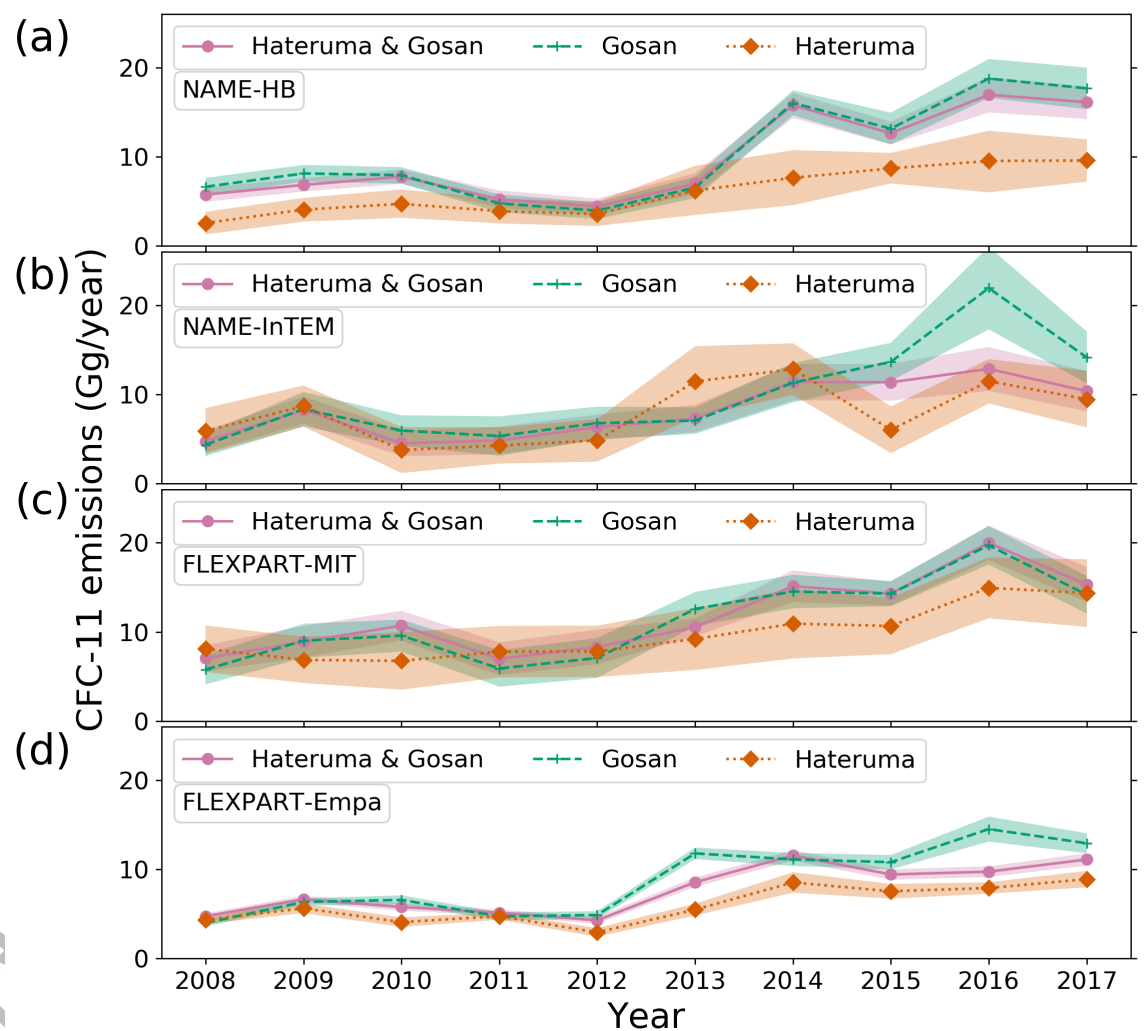

8

9 Extended Data Figure 13. Inverse emissions estimates for eastern mainland China derived

10 using only the Gosan CFC-11 measurements (green dashed line with crosses), only the

11 Hateruma (red dotted line with diamonds) CFC-11 measurements, or using both

12 measurement records in the inversion analysis (pink solid line with circles). The estimates 
1 shown use the (a) NAME-HB, (b) NAME-InTEM, (c) FLEXPART-MIT and (d)

2 FLEXPART-Empa inversion techniques.

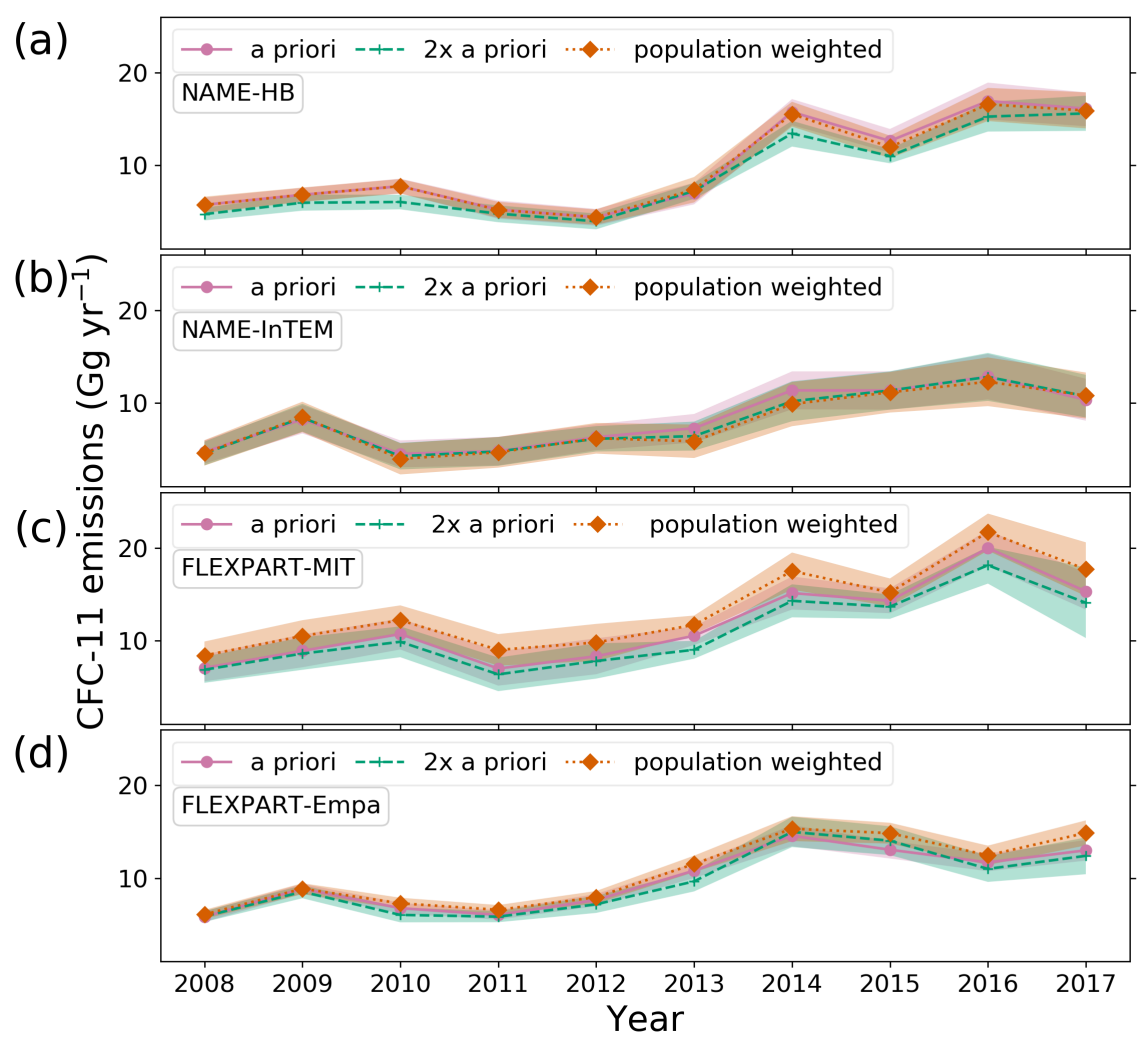

3

4 Extended Data Figure 14. Derived emissions for eastern mainland China for different a priori

5 emission magnitudes and different spatial distributions. The inversions are as described in

6 Methods ("a priori" - pink solid with circles), with a priori emissions twice as high (" $2 \mathrm{x}$ a

7 priori" - green dashed line with crosses), or the same magnitude but distributed in space

8 according to population density ("population weighted" - red dotted line with diamonds).

9 Figures show the estimates using the (a) NAME-HB, (b) NAME-InTEM, (c) FLEXPART-

10 MIT and (d) FLEXPART-Empa inversion methods. 


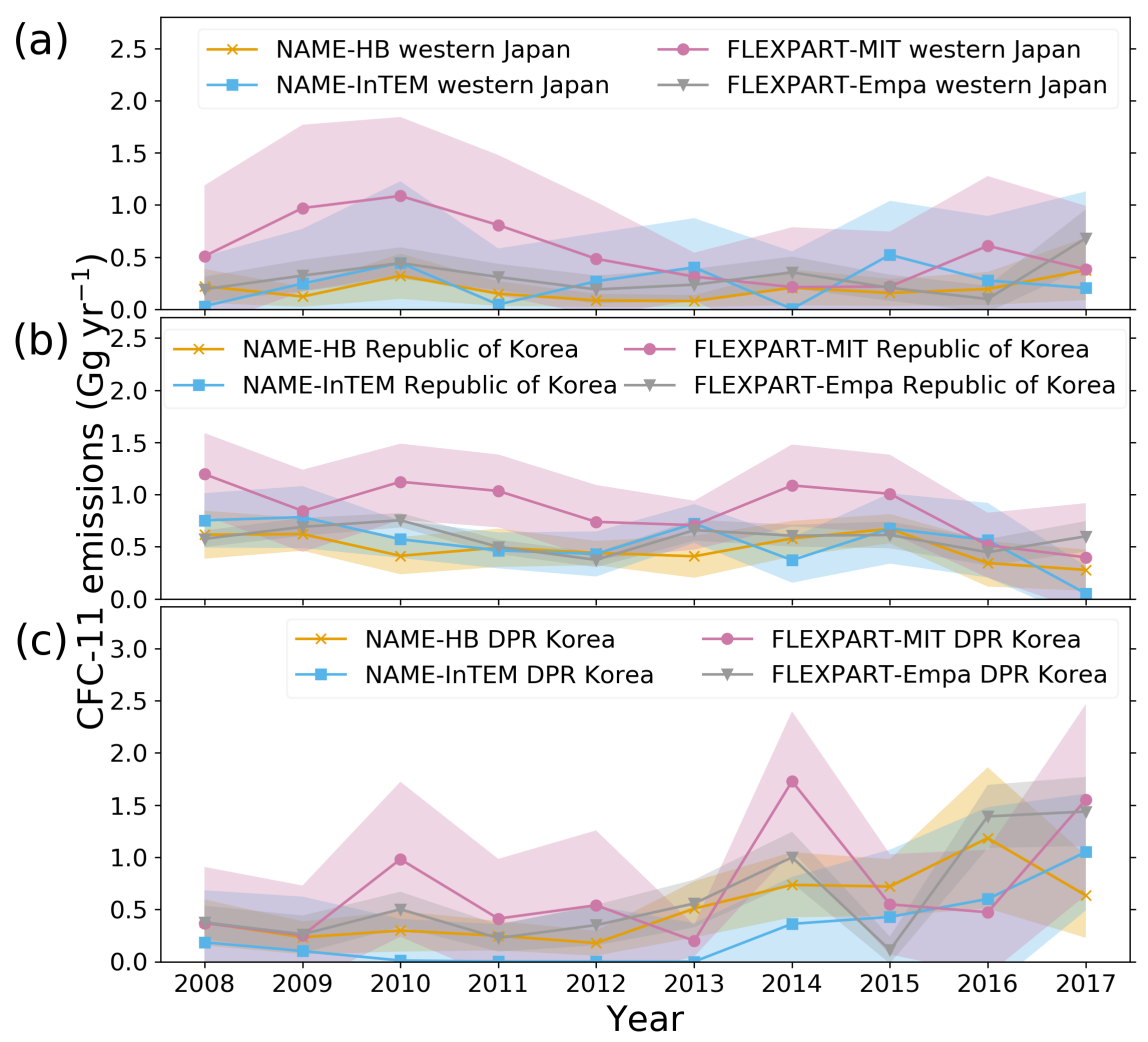

1

2 Extended Data Figure 15. Top-down emissions estimates for (a) western Japan, (b) the

3 Republic of Korea and (c) the Democratic People's Republic of Korea using the NAME-HB

4 (yellow exes), NAME-InTEM (blue squares), FLEXPART-MIT (pink circles) and

5 FLEXPART-Empa (grey inverted triangles) inverse frameworks described in Methods. See

6 Extended Data Figure 2 for the definition of the western Japan region. All lines and symbols

7 are the a posteriori mean and the shading shows the one standard deviation uncertainty. 

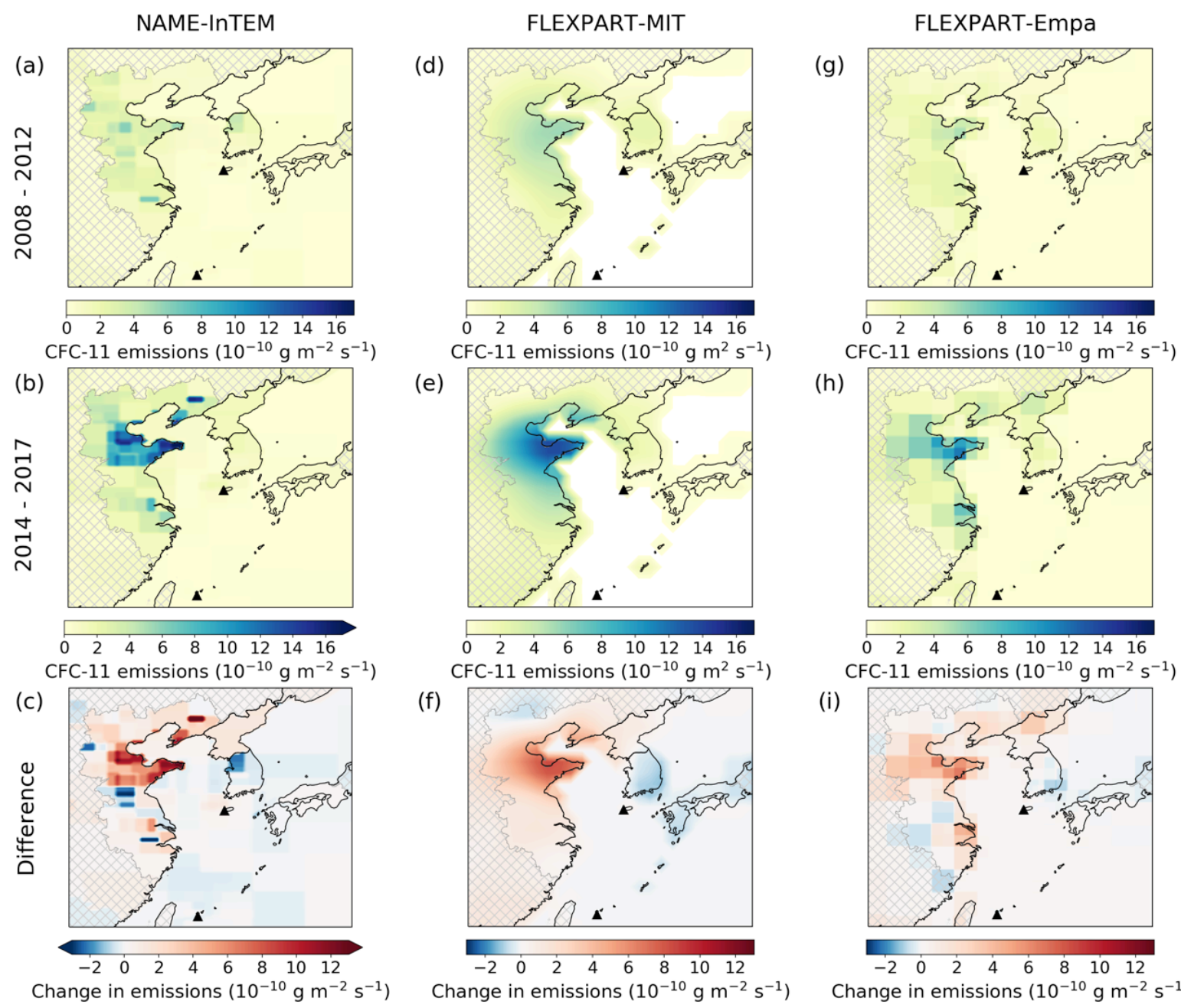

(e)
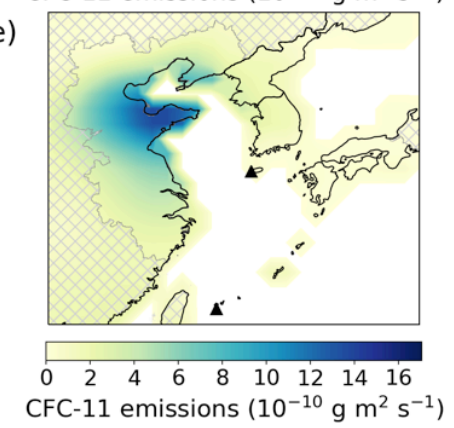

(f)

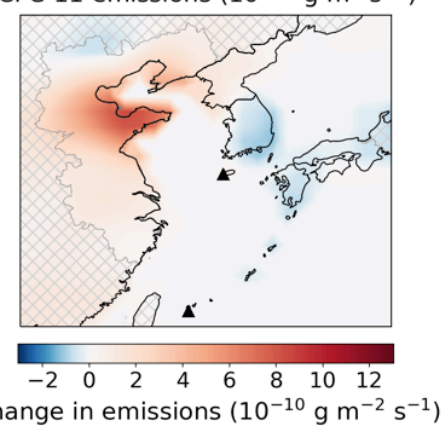

(h)
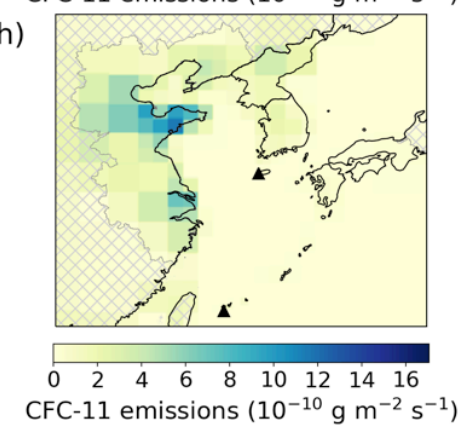

(i)

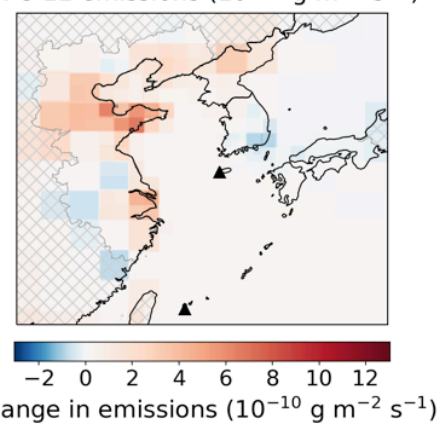

1

2 Extended Data Figure 16. Mean CFC-11 emission fluxes and difference maps, similar to

3 Figure 3, for NAME-InTEM (a-c), FLEXPART-MIT (d-f) and FLEXPART-Empa (g-i) using

4 the inversion framework described in the Methods. Figures (a), (d) and (g) show the average

5 spatial emissions for the period 2008-2012; (b), (e) and (h) show the average spatial

6 emissions for the period 2014-2017; and (c), (f) and (i) show the difference in emissions from

7 the 2008-2012 period to the 2013-2017 period, using NAME-InTEM, FLEXPART-MIT and

8 FLEXPART-Empa respectively. 


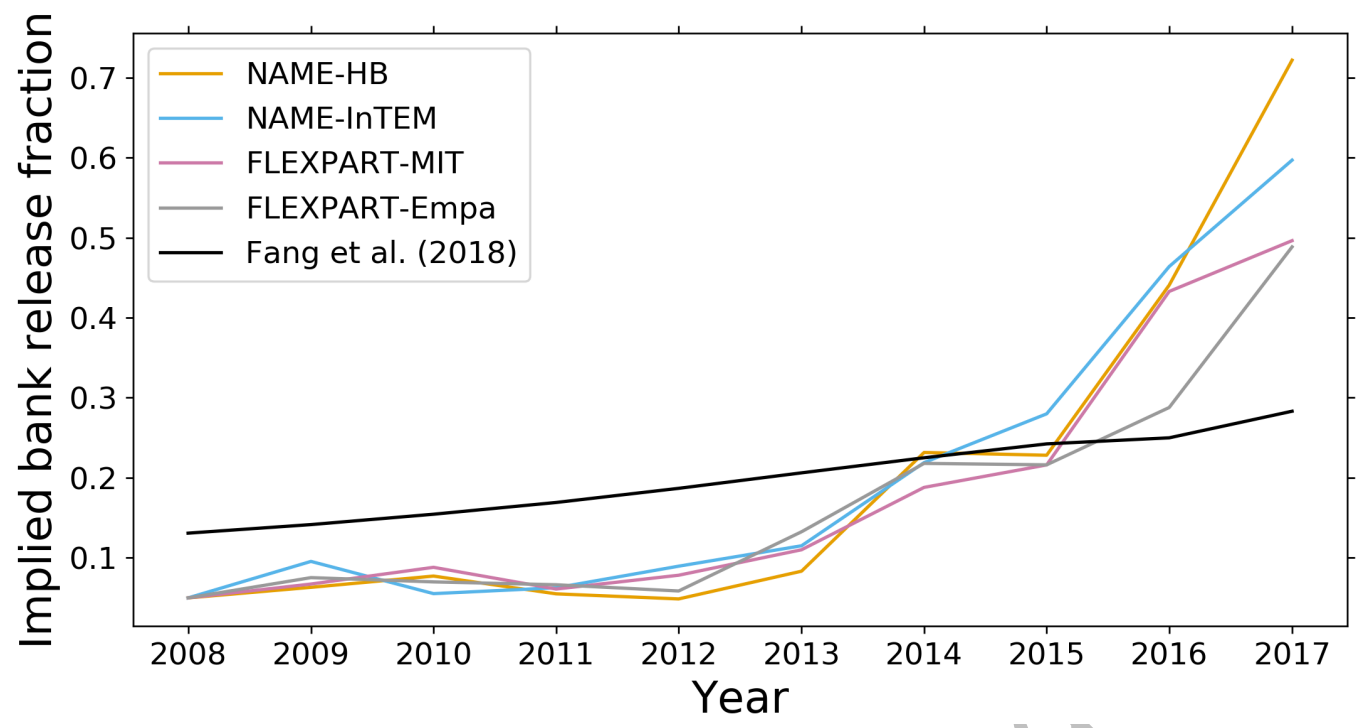

2

3 Extended Data Figure 17. The implied bank release fraction of CFC-11 from the eastern

4 mainland China region defined in Extended Data Figure 2, if there is no new production. This

5 calculation assumes that the 2008 emissions estimate are $5 \%$ of the bank size (estimated to be

6 toward the upper limit of expected bank fractional release rate $\left.{ }^{3}\right)$. The coloured lines show the

7 implied bank release fraction for each year after 2008, required to sustain the mean $a$

8 posteriori emissions from the NAME-HB (yellow), NAME-InTEM (blue), FLEXPART-MIT

9 (pink) and FLEXPART-Empa (grey) inversions. The black line shows the bank release

10 fraction from a bottom-up study for the whole of China ${ }^{7}$.

11

12

13

14 


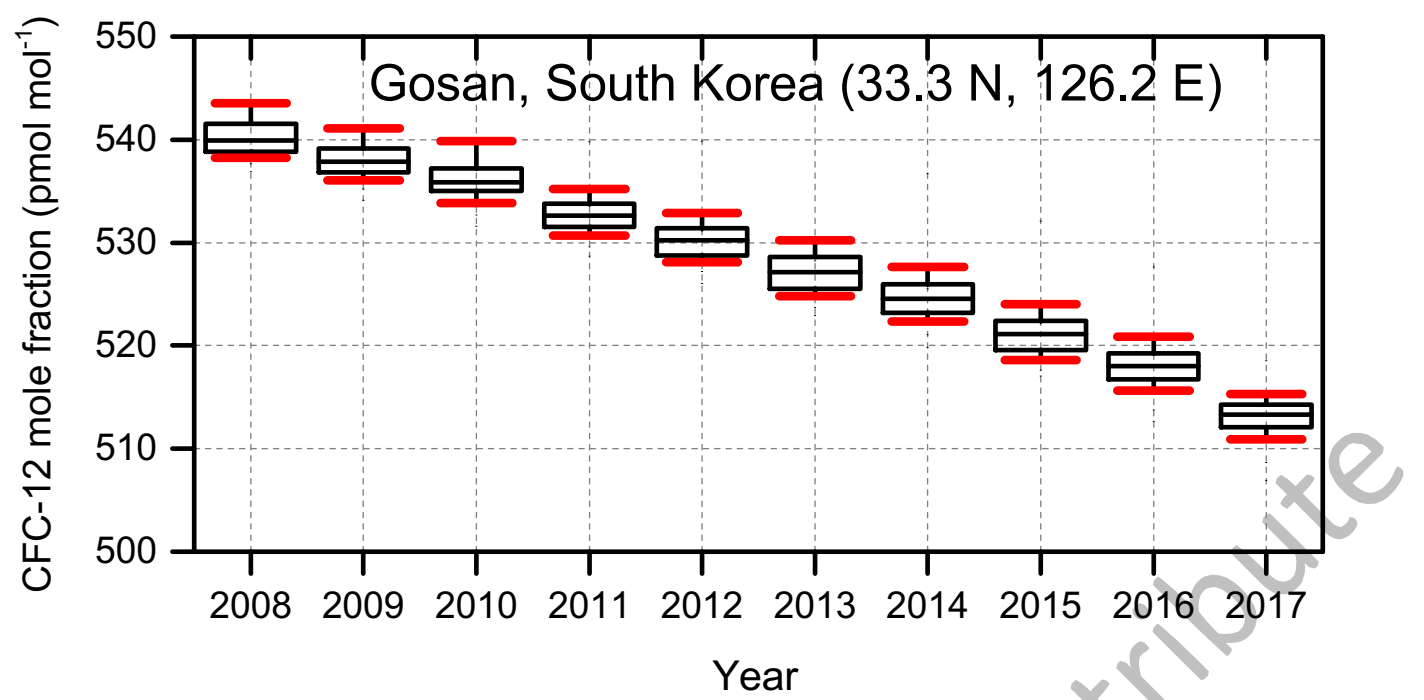

1

2 Extended Data Figure 18. Box and whisker plot of CFC-12 mole fraction at the Gosan

3 measurement site. Boxes indicate the $25^{\text {th }}$ and $75^{\text {th }}$ percentiles of the (approximately 2-

4 hourly) measurement data in a given year, and the median mole fraction is shown by the line

5 inside each box. The whiskers show the $10^{\text {th }}$ and $90^{\text {th }}$ percentiles. The lower percentiles are

6 typically representative of baseline mole fractions, and the difference between the lower and

7 higher percentiles indicates the magnitude of the above-baseline events due to the

8 interception of air masses containing recently emitted CFC-12.

9

10 